\title{
A Jacobian-free Edged-based Galerkin Formulation for Compressible Flows
}

\author{
Song Gao ${ }^{1}$, Wagdi G. Habashi ${ }^{2}$ \\ CFD Laboratory McGill University, Montreal, QC, H3A 2S6, Canada \\ Dario Isola ${ }^{3}$ and Guido S. Baruzzi ${ }^{4}$ \\ ANSYS, Inc., Montreal, QC, H3A 2M7, Canada \\ Marco Fossati ${ }^{5}$ \\ University of Strathclyde, Glasgow, G1 1XJ, UK
}

\begin{abstract}
A parallel formulation of a Jacobian-free all Mach numbers solver on unstructured hybrid meshes is proposed. The Finite Element formulation is edge-based with flow stabilization obtained with either AUSM+-up or Roe scheme. The linear system is solved via a Jacobian-Free Newton-Krylov (JFNK) method with Lower-Upper Symmetric GaussSeidel (LU-SGS) used as matrix-free preconditioner. The traditional formulation of LU-SGS is enriched by including the contributions from viscous fluxes and boundary conditions. The accuracy and efficiency of the proposed approach are demonstrated over cases ranging from low to high Mach numbers: subsonic flow over the Trap Wing, transonic flow over the ONERA M6 wing, supersonic flow over a sphere, supersonic flow over a waverider and finally hypersonic flow over a sphere.
\end{abstract}

\section{Introduction}

The

he adoption of a unified approach for the numerical study of aircraft aerodynamics over a wide range of Mach numbers is at the basis of the design of next-generation high-performance civil transport aircraft. In order to meet demands in terms of reduced fuel consumptions, sustainability and safety, it is necessary to maintain high aero-

\footnotetext{
${ }^{1}$ Doctoral candidate, CFD Laboratory, Department of Mechanical Engineering, 688 Sherbrooke Street West, AIAA Member.

${ }^{2}$ Professor and Director, CFD Laboratory, Department of Mechanical Engineering, 688 Sherbrooke Street West, AIAA Fellow.

${ }^{3}$ Senior Software Developer, ANSYS, Inc., 680 Sherbrooke Street West, AIAA Member.

${ }^{4}$ Technical Fellow, ANSYS, Inc., 680 Sherbrooke Street West, AIAA Member.

${ }^{5}$ Lecturer, Department of Mechanical and Aerospace Engineering, 75 Montrose Street, AIAA Member.
} 
thermodynamic efficiency over diverse flight and physics regimes, ranging from low Mach numbers during take-off and landing, up to supersonic and hypersonic cruise conditions. The accurate prediction of shear stresses, heat fluxes and temperature distributions on the surface of the aircraft are crucial to achieve these objectives throughout the entire flight envelope. The formulation of a single scheme for subsonic to hypersonic speeds is hampered by the increasing dominance of convection and high-temperature effects that characterize these regimes. Strong shock waves, chemical reactions, thermal non-equilibrium, and the potential occurrence of magneto-gasdynamics effects make the design of an efficient numerical approach rather complex. Despite the efforts put forward by researchers over the years, a high Technology Readiness Level (TRL) consolidated multiphysics approach has yet to be achieved [1-3].

A complex system of conservation equations modeling chemical and thermal non-equilibrium demands a computational framework able to solve stiff problems characterized by a large number of chemical species. At high Damköhler numbers, the timescales of the chemistry are typically much faster than the ones of the flow. On the numerical side, this has led researchers to prefer implicit methods to explicit ones to overcome time step restrictions due to stability issues [4]. For steady-state problems, the governing equations are often solved implicitly through an exact or inexact Newton-Raphson procedure. At every Newton step, the residual function is linearized and the update is computed by solving a linear system. Compared to explicit methods, traditional implicit algorithms require two additional operations: the analytical differentiation of the residual and the assembly of the Jacobian matrix. For high enthalpy flows, practical problems arise that can potentially threaten the efficiency of implicit strategies. The first step becomes increasingly difficult as the physics is enriched to include high temperature thermodynamic effects. The second step is memory-bound by the number of species. An analytical differentiation of the Jacobian is adopted in the FIN-S solver of Kirk et al. [5]. Numerical approximation of the Jacobian can overcome some of the aforementioned problems, but at the expense of increasing computational time. Lani et al. [6] have developed a solver that numerically computes each column of the Jacobian by forward finite differences and then stores the Jacobian. The resulting scheme is slower than the one obtained with analytic differentiation, and still requires significant memory. In the numerical solution of high-Mach flows by implicit methods, the memory footprint of the Jacobian can be very large and the number of degrees of freedom per node increases with the number of species, making it more important to limit memory requirements. One possibility is the line-implicit relaxation method used in the solver LeMANS [7]. It adopts a line search method that finds the normal direction of the wall and a 
renumbering algorithm that reorders the elements, and then the off-tridiagonal terms of the linearized system are moved to the right-hand-side, whereas the left-hand-side tridiagonal system is solved by a point-iterative method without storing the Jacobian. In the Jacobian-Free Newton-Krylov (JFNK) method adopted in this work the Jacobian-vector product is approximated by a Fréchet derivative, which circumvents the need to store the full Jacobian, and a Krylov subspace method is used to solve the linear system [8].

The effectiveness of Krylov subspace linear system solvers depends on good preconditioners. The widely-used Incomplete Lower-Upper (ILU) factorization not only requires storing the system matrix but also its factorization, thus doubling the memory footprint. To the best of the authors' knowledge, three alternate options are available. The first one is the Jacobi preconditioner, which can be implemented in a Jacobian-free fashion. This method only requires the diagonal of the Jacobian. While it seems numerically advantageous, a large number of linear iterations might be required for convergence. The second alternative is the multiplicative-additive Schwarz preconditioned inexact Newton [9] approach, which is a nonlinear Jacobian-free preconditioning method designed to overcome unbalanced nonlinearities coming from different ranges of time and spatial scales, such as shock waves and reaction fronts, but only two-dimensional incompressible flow test cases have been presented in the reference. The last alternative, adopted in this work, is LU-SGS [4]. It was originally developed as a solver for inviscid flows on structured grids, but has recently been extended to viscous flows and unstructured meshes [10] [11]. In LU-SGS, the implicit operator is simplified by introducing a Roe-type flux approximation that replaces the Roe matrix with its spectral radius, consequently only the diagonal part of the Jacobian is stored and the products of the off-diagonal terms and the solution update are approximated by a Fréchet derivative through a forward and a backward sweep. However, in the original LU-SGS, the viscous Jacobian is identified as a scalar in the spectral radius and the contributions from boundary conditions are omitted. In this work, the viscous Jacobian is taken into account by computing it on the fly during the two sweeps. Riemann, supersonic outlet, slip wall and non-slip wall boundary conditions are included in the computation of the Jacobian.

This article is organized as follows: Section II will illustrate the governing equations, while Section III will present the numerical formulation and Section IV will present some numerical results for validation. The test cases for the present work are limited to non-reactive flows in thermal equilibrium. This is a necessary step to assess the characteristics of the method before introducing the more complex non-equilibrium effects and the corresponding numerical formulation. 


\section{Governing Equations}

Let us recall for completeness the equations governing unsteady compressible viscous flows in conservative form $[12,13]$

$$
\frac{\partial \mathrm{Q}}{\partial \mathrm{t}}+\nabla \cdot\left(\mathbf{F}^{\mathrm{A}}(\mathrm{Q})-\mathbf{F}^{\mathrm{V}}(\mathrm{Q}, \nabla \mathrm{Q})\right)=0
$$

where $\mathbf{Q}$ is the vector of conservative variables, i.e. $\mathbf{Q}=\left[\rho, \rho \mathbf{V}, \rho \mathrm{e}^{\mathrm{t}}\right] . \rho \mathbf{V}$ is the momentum vector and $\mathrm{e}^{\mathrm{t}}$ is the total energy per unit mass and it is defined as the sum of the internal and kinetic energy. $\mathbf{F}^{A}$ and $\mathbf{F}^{\vee}$ are the inviscid and viscous fluxes, respectively. The inviscid fluxes in Cartesian coordinates are

$$
F_{x}^{A}=\left\{\begin{array}{c}
\rho u \\
\rho u^{2}+P \\
\rho u v \\
\rho u w \\
u\left(\rho e^{t}+P\right)
\end{array}\right\} ; \quad F_{y}^{A}=\left\{\begin{array}{c}
\rho v \\
\rho u v \\
\rho v^{2}+P \\
\rho v w \\
v\left(\rho e^{t}+P\right)
\end{array}\right\} ; \quad F_{z}^{A}=\left\{\begin{array}{c}
\rho w \\
\rho u w \\
\rho v w \\
\rho w^{2}+P \\
w\left(\rho e^{t}+P\right)
\end{array}\right\}
$$

The viscous fluxes in Cartesian coordinates are

$$
F_{x}^{v}=\left\{\begin{array}{c}
0 \\
\tau_{x x} \\
\tau_{x y} \\
\tau_{x z} \\
v_{\tau_{x}}
\end{array}\right\} ; \quad F_{y}^{v}=\left\{\begin{array}{c}
0 \\
\tau_{y x} \\
\tau_{y y} \\
\tau_{y z} \\
v_{\tau_{y}}
\end{array}\right\} ; \quad F_{z}^{v}=\left\{\begin{array}{c}
0 \\
\tau_{z x} \\
\tau_{z y} \\
\tau_{z z} \\
\mathbf{v} \tau_{z}
\end{array}\right\}
$$

Newton's law for the stress tensor and the Stokes hypothesis are adopted and a calorically perfect ideal gas is assumed.

\section{Numerical Methods}

\section{A. Edge-based Stable Finite Element Discretization}

A Finite Element edge-based assembly [14] is adopted to allow the application of either AUSM ${ }^{+}$up [15] or Roe [16] scheme to stabilize the advection terms. The edge-based assembly easily handles hybrid meshes with a unique data structure [17] and it is computationally more efficient than the traditional element-based one [18]. For steadystate flows, the weak-Galerkin formulation of Eq. (II.1) is adopted: 


$$
\sum_{e \in \Psi_{i} V_{e}} \nabla W_{i} \cdot\left(F^{A}-F^{V}\right) d \forall+\sum_{e G_{i}} \int_{i} W_{i} \cdot\left(F^{A}-F^{V}\right) d A=0
$$

where $W_{i}$ is the $i$-th linear Lagrangian test function, $E_{i}$ and $F_{i}$ are the set of elements/boundary faces sharing the i-th vertex. For ease of notation, the contribution from boundary conditions will be ignored from this point on. The first term of the above equation can be assembled in an edge-based fashion as [19]:

$$
\sum_{j \in K_{i}} \eta_{i j} \cdot \frac{F_{j}^{A}+F_{i}^{A}}{2}-\sum_{j \in K_{i}} x_{i j} \cdot \frac{F_{j}^{A}-F_{i}^{A}}{2}+\sum_{e \in \Psi_{i}} \int_{e} \nabla W_{i} \cdot F^{V} d V=0
$$

where $\mathrm{K}_{\mathrm{i}}$ is the set of nodes connected to the $\mathrm{i}$-th node via an edge. The edge coefficients are defined as

$$
\eta_{i j}=\sum_{e \in \Psi_{i}}\left(\int_{V_{e}} W_{i} \nabla W_{j} d V-\int_{V_{e}} W_{j} \nabla W_{i} d V\right) \quad \text { and } \quad \chi_{i j}=\sum_{e \Psi_{i} A} \int_{A} W_{i} W_{j} n d A
$$

where the coefficient $\chi_{i j}$ is defined only for boundary edges. Another second order edge coefficient tensor $\mathrm{d}_{\mathrm{ij}}$ can be defined as

$$
d_{i j}=\sum_{e \in \Psi_{i}} \int_{e} \nabla W_{i} \nabla W_{j} d V, \quad d_{i j}^{s}=\frac{d_{i j}+\left(d_{i j}\right)^{\top}}{2}, \quad d_{i j}^{a}=\frac{d_{i j}-\left(d_{i j}\right)^{\top}}{2}
$$

To provide stabilization for advection-dominated flows, the vector of inviscid fluxes $\mathbf{F}^{\mathrm{A}}$ is replaced with a numerical counterpart, $\Phi^{\text {num }}$ evaluated at the edge's midpoint, i.e.

$$
\sum_{j \in \varepsilon_{i}} \Phi^{n u m}\left(Q_{i}, Q_{j}, \eta_{i j}\right)-\sum_{j \in \varepsilon_{i}} \chi_{i j} \cdot \frac{F_{j}^{A}-F_{i}^{A}}{2}+\sum_{e \in \Psi_{i}} \int_{V_{e}} \nabla W_{i} \cdot F^{\vee} d V=0
$$

The numerical inviscid fluxes for $\Phi^{\text {num }}$ can be either $\mathrm{AUSM}^{+}$-up or Roe fluxes, nonlinear functions of the nodal variables and of the edge coefficients. The boundary-edge term of Eq. (III.5) can be seen as a "correction" factor, since it is proportional to the difference of fluxes along the edge. Following [14], it is left untouched and no additional dissipation is introduced for boundary edges. The numerical scheme is made second-order by adopting a MUSCL [20] reconstruction of the primitive variables at the edges' midpoint and standard 1D slope limiters [21, 22]. The viscous fluxes are discretized with the continuous Galerkin approximation and assembled in an edge-based fashion, naturally resulting in a second-order representation [14]. 


\section{B. Jacobian-free Newton-Krylov Solver}

The JFNK [8] strategy is introduced by making use of a pseudo-transient continuation method where the original steady problem is transformed into a pseudo-unsteady one [23], i.e.

$$
r^{n}=L \frac{Q^{n}-Q^{n-1}}{\Delta \tau^{n}}+R\left(Q^{n}\right)=0, \quad n=1,2, \ldots
$$

where $L$ is the lumped mass matrix, $R$ is the left-hand-side of Eq. (III.5). The pseudo-time step $\Delta \tau^{n}$ is chosen to locally satisfy the CFL stability condition for linear advection problems and is in general increased as the solution progresses. At each iteration of the Newton procedure, the solution update is computed as

$$
\left[\frac{L}{\Delta \tau} \mathrm{I}+\frac{\partial \mathrm{R}}{\partial \mathrm{Q}}\right]^{\mathrm{n}} \Delta \mathrm{Q}=-\mathrm{R}\left(\mathrm{Q}^{\mathrm{n}}\right)
$$

Note that small values of $\Delta \tau^{n}$ increase the diagonal dominance of the system matrix, making it easier to invert. As $\Delta \tau^{\mathrm{n}}$ increases, the first term of Eq. (III.7) gradually vanishes and the pseudo-unsteady problem reverts to the standard Newton's method. The above linear system is solved by means of the Flexible Generalized Minimal Residual method (FGMRES) [24]. FGMRES is an iterative solver for non-symmetric linear systems where the preconditioner can vary at each iteration. The convergence of FGMRES depends on the condition number of the matrix; preconditioning techniques can be enforced to cluster the eigenvalues of the matrix and to improve the convergence of the linear system. Defining

$$
A=\left[\frac{L}{\Delta \tau} I+\frac{\partial R}{\partial Q}\right]^{n} \text { and } \Delta P=M \Delta Q
$$

and the preconditioner by $\mathrm{M}$, the right preconditioning results

$$
\left(A M^{-1}\right)(\Delta P)=-R
$$

FGMRES only accesses the matrix through matrix-vector multiplications, therefore it is not necessary to form and store the matrix A. In the framework of Jacobian-free methods, the product of the preconditioned system matrix with the preconditioned solution is replaced by a Fréchet derivative, i.e. 


$$
\begin{aligned}
\left(\mathrm{AM}^{-1}\right)(\Delta \mathrm{P}) & =\left[\frac{L}{\Delta \tau}+\frac{\partial \mathrm{R}}{\partial \mathrm{Q}}\right]^{\mathrm{n}} \mathrm{M}^{-1} \Delta \mathrm{P} \\
& ; L \frac{\Delta \mathrm{Q}}{\Delta \tau}+\frac{\mathrm{R}\left(\mathrm{Q}^{\mathrm{n}}+\varepsilon \mathrm{M}^{-1} \Delta \mathrm{P}\right)-\mathrm{R}\left(\mathrm{Q}^{\mathrm{n}}\right)}{\varepsilon}
\end{aligned}
$$

where $\varepsilon$ is a suitably-chosen small number [25] defined as

$$
\varepsilon=\text { error_rel } \cdot \frac{\sqrt{1+\left\|Q^{n}\right\|}}{\|\Delta Q\|}
$$

with error_rel being the square root of the machine precision.

Note that the JFNK methods require one evaluation of the residual function R at each FGMRES iteration, while traditional methods that explicitly form the Jacobian require one matrix-vector product per FGMRES iteration. For a problem of millions of unknowns, one evaluation of the residual function typically requires more time than one matrix-vector multiplication, indicating that overall JFNK might be slower than traditional methods. It is also worth mentioning that the JFNK methods only require the evaluation of $R$, but not its derivatives, which enables the computation of the inviscid contribution to the Jacobian matrix through the MUSCL-reconstructed state with slopelimiter, or a simple piecewise constant reconstruction of the solutions.

\section{Lower-Upper Symmetric-Gauss-Seidel (LU-SGS) Preconditioner}

In order to obtain a truly Jacobian-free method, the preconditioning step must also be Jacobian-free. LU-SGS is an efficient iterative solver specifically designed for advection-dominated flows that does not require storing the Jacobian. Following [4], LU-SGS is applied by solving

$$
A \Delta Q=-R\left(Q^{n}\right)
$$

through a forward and backward sweep, namely

$$
\begin{aligned}
& (D+L) \Delta Q^{*}=-R \\
& (D+U) \Delta Q=D \Delta Q^{*}
\end{aligned}
$$

where $\mathrm{A}$ is the sum of the pseudo-time contribution and the Jacobian of the approximate residual function 


$$
\mathrm{A}=\frac{L}{\Delta \mathrm{t}} \mathrm{I}+\frac{\partial \mathrm{R}}{\partial \mathrm{Q}}
$$

and $\mathrm{D}, \mathrm{U}$ and $\mathrm{L}$ are the diagonal, upper and lower parts of matrix $\mathrm{A}$, respectively.

To compute A cost-effectively, the approximate residual function $\mathrm{R}$ is defined after discarding the second term in Eq. (III.5), neglecting the boundary contribution of the viscous fluxes and replacing the inviscid fluxes with a simpler approximate one

$$
\begin{aligned}
& 647^{\mathrm{A}} 48 \quad 647^{\mathrm{V}} 48 \quad \text { G55\$ }
\end{aligned}
$$

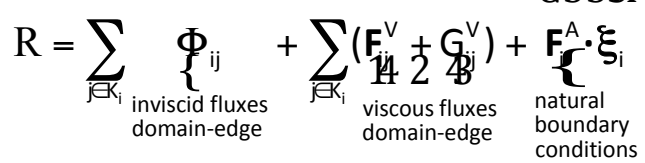

where $\xi_{i}$ is the nodal boundary coefficient, $\mathbf{F}_{\mathrm{ij}}{ }^{\mathrm{V}}$ is the viscous flux in momentum equation, $\mathrm{G}_{\mathrm{ij}}{ }^{\mathrm{V}}$ is the viscous flux in energy equation and $\mathrm{R}^{\mathrm{A}}, \mathrm{R}^{\mathrm{V}}$ and $\mathrm{R}^{\partial}$ are the contributions from inviscid and viscous fluxes for the domain edges and the natural boundary conditions, respectively. The approximate residual function R adopted in this work is different from Luo's [4]. In his work, $\mathrm{R}^{\partial}$ is ignored and $\mathrm{R}^{\mathrm{V}}$ is replaced by a scalar appearing in $\mathrm{R}^{\mathrm{A}}$.

$\Phi_{\mathrm{ij}}$ is a simplified version of $\Phi^{\text {num }}$, i.e.

$$
\Phi_{i j}=\Phi\left(Q_{i}, Q_{j}, \eta_{i j}\right)=\frac{F^{A}\left(Q_{i}\right)+F^{A}\left(Q_{j}\right)}{2} \cdot \eta_{i j}-\frac{\left|\Lambda_{i j}\right|}{2}\left(Q_{j}-Q_{i}\right)
$$

where the spectral radius

$$
\left|\Lambda_{\mathrm{ij}}\right|=\left|\mathbf{V}_{\mathrm{ij}} \cdot \boldsymbol{\eta}_{\mathrm{ij}}\right|+\left|\boldsymbol{\eta}_{\mathrm{ij}}\right| \mathrm{a}_{\mathrm{ij}}
$$

is used to approximate the artificial diffusion operator. In Eq. (III.16) the velocity $\mathbf{V}_{\mathrm{ij}}$ and the sound speed $\mathbf{a}_{\mathrm{ij}}$ are evaluated at a Roe-averaged state [16] between the two nodes.

Neglecting boundary terms and addressing the Jacobian of the inviscid fluxes $R^{A}$ for the domain edges, Eq. (III.14) is differentiated with respect to $Q_{i}$ and $Q_{j}$, and then the upper matrix $U_{i j}^{A}$, the lower matrix $L_{i j}^{A}$ and the diagonal matrix $\mathrm{D}_{\mathrm{ij}}^{\mathrm{A}}$ are 


$$
\mathrm{U}_{\mathrm{ij}}^{\mathrm{A}}=\frac{1}{2}\left[\frac{\partial \mathrm{F}_{\mathrm{j}}^{\mathrm{A}} \cdot \boldsymbol{\eta}_{\mathrm{ij}}}{\partial \mathrm{Q}_{\mathrm{j}}}-\left|\Lambda_{\mathrm{ij}}\right| \mathrm{I}\right], \mathrm{L}_{\mathrm{ij}}^{\mathrm{A}}=-\frac{1}{2}\left[\frac{\partial \mathrm{F}_{\mathrm{j}}^{\mathrm{A}} \cdot \boldsymbol{\eta}_{\mathrm{ji}}}{\partial \mathrm{Q}_{\mathrm{j}}}+\left|\Lambda_{\mathrm{ij}}\right|\right], \mathrm{D}_{\mathrm{ii}}^{\mathrm{A}}=\left[\frac{L_{\mathrm{i}}}{\Delta \mathrm{t}_{\mathrm{i}}}+\sum_{\mathrm{j}=1}^{\mathrm{N}_{\mathrm{e}}} \frac{\left|\Lambda_{\mathrm{ij}}\right|}{2}\right] \mathrm{I}
$$

Note that the zero-sum property of the edge coefficients for domain nodes has been used and the pseudo-time contribution has been added to the diagonal matrix. As a result, the $5 \times 5$ matrix located on the diagonal block is replaced by a scalar, thus making its inversion a trivial operation.

With this strategy $U^{A}, L^{A}$ and $D^{A}$ must be computed and explicitly stored, but this can be avoided by introducing a Jacobian-free approximation of the matrix-vector product as

$$
\frac{\partial F_{j}^{A} \cdot \eta_{i j}}{\partial Q_{j}} \Delta Q_{j}^{*}=\frac{F^{A}\left(Q_{j}+\varepsilon \Delta Q_{j}^{*}\right) \cdot \eta_{i j}-F^{A}\left(Q_{j}\right) \cdot \eta_{i j}}{\varepsilon}
$$

where $\varepsilon$ is defined in Eq. (III.10).

To address the Jacobian of $\mathrm{R}^{\mathrm{V}}, \mathrm{F}_{\mathrm{ij}}{ }^{\mathrm{V}}$ and $\mathrm{G}_{\mathrm{ij}}{ }^{\mathrm{V}}$ are discretized with the standard continuous Galerkin approximation and assembled in an edge-based fashion, as in [14]. The upper matrix $\mathrm{U}_{\mathrm{ij}}{ }^{\mathrm{V}}$, the lower matrix $\mathrm{L}_{\mathrm{ij}}{ }^{\mathrm{V}}$ and the diagonal matrix $\mathrm{D}_{\mathrm{ij}}{ }^{\mathrm{v}}$ are

$$
\begin{aligned}
D_{i i}^{V}= & I_{5,3} \cdot\left[-\sum_{i<j}\left(A_{i j}^{V, s}+A_{i j}^{V, a}\right)-\sum_{j<i}\left(A_{j i}^{V, s}-A_{j i}^{V, a}\right)\right] \frac{\partial \mathbf{V}_{i}}{\partial Q_{i}} \\
& +I_{5,1} \cdot\left[-\sum_{i<j} \mathbf{V}_{i j} \cdot\left(A_{i j}^{V, s}+A_{i j}^{V, a}\right)-\sum_{j<i} \mathbf{V}_{i j} \cdot\left(A_{j i}^{V, s}-A_{j i}^{V, a}\right)\right] \frac{\partial \mathbf{V}_{i}}{\partial Q_{i}} \\
U_{i j}^{V}= & I_{5,3} \cdot\left(A_{i j}^{V, s}+A_{i j}^{V, a}\right) \frac{\partial \mathbf{V}_{j}}{\partial Q_{j}}+I_{5,1} \cdot \mathbf{V}_{i j} \cdot\left(A_{i j}^{V, s}+A_{i j}^{V, a}\right) \cdot \frac{\partial \mathbf{V}_{j}}{\partial Q_{j}} \\
L_{i j}^{V}= & I_{5,3} \cdot\left(A_{j i}^{V, s}-A_{j i}^{V, a}\right) \frac{\partial \mathbf{V}_{j}}{\partial Q_{j}}+I_{5,1} \cdot \mathbf{V}_{i j} \cdot\left(A_{j i}^{V, s}-A_{j i}^{V, a}\right) \cdot \frac{\partial \mathbf{V}_{j}}{\partial Q_{j}}
\end{aligned}
$$

where the "s" and "a" superscripts indicate the symmetric and anti-symmetric part of a tensor, i.e.

$$
A_{i j}^{V, s}=\mu_{i j} \operatorname{tr}\left(d_{i j}\right) l+\left(\mu_{i j}+\lambda_{i j}\right) d_{i j}^{s}, \quad A_{i j}^{V, a}=\left(\lambda_{i j}-\mu_{i j}\right) d_{i j}^{a}
$$

The transformation matrices are 


$$
\mathrm{I}_{5,3}=\left[\begin{array}{lllll}
0 & 1 & 0 & 0 & 0 \\
0 & 0 & 1 & 0 & 0 \\
0 & 0 & 0 & 1 & 0
\end{array}\right]^{T} \quad \text { and } \quad \mathrm{I}_{5,1}=\left[\begin{array}{lllll}
0 & 0 & 0 & 0 & 1
\end{array}\right]^{T}
$$

The Jacobian of $\mathrm{R}^{\partial}$ only contributes to the diagonal matrix, i.e.

$$
\mathrm{D}_{\mathrm{ii}}^{\partial}=\frac{\partial \mathbf{F}_{\mathrm{i}}^{\partial} \cdot \boldsymbol{\xi}_{\mathrm{i}}}{\partial \mathrm{Q}_{\mathrm{i}}}
$$

where the expression of $\mathbf{F}_{\mathrm{i}}^{\partial}$ depends on the type of boundary conditions. In the case of slip wall and symmetry planes, $\mathbf{F}_{i}^{\partial} \boldsymbol{\xi}_{i}=\left[0, P_{i} \xi_{i}, 0\right]$. In the case of Riemann invariant boundary conditions, $\mathbf{F}_{i}{ }^{\partial} \boldsymbol{\xi}_{i}=\mathbf{F}_{i}^{A}\left(Q_{\text {riemann }}\right) \cdot \xi_{i}$, where $\mathrm{Q}_{\text {riemann }}$ is computed by solving the Riemann problem between the internal node $\mathrm{i}$ and the free-stream. In the case of supersonic outlet, $\mathbf{F}_{\mathrm{i}}^{\partial} \cdot \boldsymbol{\xi}_{\mathrm{i}}=\mathbf{F}_{\mathrm{i}}^{\mathrm{A}} \cdot \boldsymbol{\xi}_{\mathrm{i}} \cdot[26] \mathrm{D}^{\partial}$ can be combined with $\mathrm{D}^{\mathrm{A}}$ for further simplification

$$
\begin{aligned}
& \mathrm{D}_{\mathrm{ii}}^{\mathrm{A}+\partial}=\frac{L_{\mathrm{ii}}}{\Delta \tau} \mathrm{I}+\frac{\partial}{\partial \mathrm{Q}_{\mathrm{i}}}\left[\mathrm{F}_{\mathrm{i}}^{\partial} \cdot \xi_{\mathrm{i}}+\frac{1}{2} \sum_{\mathrm{j} \in \mathrm{K}_{\mathrm{i}}}\left(\mathrm{F}_{\mathrm{i}}^{\mathrm{A}} \cdot \boldsymbol{\eta}_{\mathrm{ij}}+\left|\lambda_{\mathrm{ij}}\right| \mathrm{Q}_{\mathrm{i}}\right)\right] \\
& =\left[\frac{L_{i i}}{\Delta \tau}+\sum_{j \in k_{i}} \frac{\left|\Lambda_{i j}\right|}{2}\right] I+\frac{\partial}{\partial Q_{i}}\left[\left(F_{i}^{\partial}-\frac{F_{i}^{A}}{2}\right) \cdot \xi_{i}+\sum_{j \in k_{i}} F_{i}^{A} \cdot\left(\frac{\eta_{i j}}{2}+\frac{\xi_{i}}{2}\right)\right] \\
& =\left[\frac{L_{\mathrm{ii}}}{\Delta \tau}+\sum_{j \in \mathrm{F}_{\mathrm{i}}} \frac{\left|\Lambda_{\mathrm{ij}}\right|}{2}\right] I+\frac{\partial}{\partial \mathrm{Q}_{\mathrm{i}}}\left[\left(\mathbf{F}_{\mathrm{i}}^{\partial}-\frac{\mathbf{F}_{\mathrm{i}}^{\mathrm{A}}}{2}\right) \cdot \boldsymbol{\xi}_{\mathrm{i}}\right]
\end{aligned}
$$

where the zero-sum property of edge coefficients has been applied. An immediate benefit is that the last term of $\mathrm{D}^{\mathrm{A}+\partial}$ is independent of the node $\mathrm{j}$.

Finally, the LU-SGS sweeps in Eq. (III.12) can be written as

$$
\begin{aligned}
& \left(D^{A+\partial}+D^{V}+L^{A}+L^{V}\right) \Delta Q^{*}=-R \\
& \left(D^{A+\partial}+D^{V}+U^{A}+U^{V}\right) \Delta Q=\left(D^{A+\partial}+D^{V}\right) \Delta Q^{*}
\end{aligned}
$$

The velocity components on the wall are zero for the non-slip wall boundary conditions, however the momentum contributions on the walls must be reset to zero after each sweep since the LU-SGS procedure may not automatically satisfy the condition. A remarkable feature of LU-SGS is that the storage of the Jacobian is not required. In this work, the PETSc implementation of FGMRES is adopted [27], while an in-house version of LU-SGS has been implemented within the PETSc framework. 


\section{Numerical Results}

The proposed numerical method has been validated over a representative range of Mach numbers, for 3D test cases. These are the subsonic flow over the Trap Wing, the transonic flow over the ONERA M6 wing, the Mach 1.93 laminar flow over a sphere, the Mach 4.0 turbulence flow over a waverider and the hypersonic flow over a sphere.

With the present JFNK implementation the inviscid contribution to the Jacobian matrix can be computed either using a simple piecewise-constant reconstruction of the solution, or a MUSCL reconstructed state with slope-limiter. The first one will be subsequently referred to as $1^{\text {st }}$ order Jacobian (JFNK-1), and the second as $2^{\text {nd }}$ order Jacobian (JFNK-2). In order to assess the performance of the JFNK solvers, the traditional method of explicitly forming the Jacobian and using block-Jacobi with ILU preconditioning is also tested. This will subsequently be referred to as the explicit Jacobian method. The explicit Jacobian method uses a simple piecewise-constant reconstruction of the solution to compute the Jacobian. Computing the explicit Jacobian using the MUSCL reconstructed states and the slope-limiter is difficult because a larger stencil is required to compute their derivatives. However, JFNK does not have such a problem because its Jacobian is numerically approximated and the derivatives are not needed.

Unless otherwise specified, all the results shown in the following sections are obtained with JFNK-2. FGMRES convergence is achieved when the relative drop in the preconditioned residual norm is below the specified tolerance of $10^{-4}$. The size of the Krylov space is 20. For the first three test cases, FGMRES is stopped when the number of FGMRES iterations exceeds 20 and the code proceeds to the next Newton step. No FGMRES restarts have been allowed except for the last two test case. Stabilization is provided by the AUSM+-up or Roe scheme with the van Albada slope limiter. The ILU preconditioner remains fixed at each FGMRES iteration, in which case the results of FGMRES are identical to those of GMRES.

\section{A. Mach 0.2 Viscous Flow past the Trap Wing}

This example is the Mach 0.2 viscous flow around the Trap Wing from the First AIAA CFD High-Lift Prediction Workshop [28]. The angle of attack is 13 degrees. The Reynolds number based on the mean aerodynamic chord (MAC) is $4.3 \times 10^{6}$ and the turbulent flow is modeled with the Spalart-Allmaras turbulence model, solved in a loosely coupled fashion. The computational grid, shown in Figure 1, consists of 8,188,411 nodes, 
$8,632,114$ tetrahedra and $13,271,131$ prisms. The surface of the wing is discretized with 446,420 triangles. The initial CFL number is 0.1 , exponentially increased to 10 in 1000 iterations.

Figure 1 and Figure 2 show the computed pressure coefficient distribution compared to the experimental data [28] at the $50 \%$ spanwise station. The agreement is good on each of the three elements. Table 1 lists the lift and drag coefficients from three numerical results against the reference values. The difference in lift coefficient between the results obtained with the JFNK-2 solver and the reference data is less than 0.036 or $1.8 \%$. The difference in drag coefficients is less than 10 counts or $0.4 \%$. Figure 1 shows the contours of pressure coefficient and Figure 3 shows the contours of shear stress magnitude. The shear stress magnitude does not reach zero on the main element and the flap, which means that no flow separation occurs.

Figure 4 shows the convergence curves for explicit Jacobian, JFNK-1 and JFNK-2 solvers. In the graphs, time is defined as the average time per FGMRES iteration for JFNK-2, which is 6.15 seconds for this test case. In terms of Newton iterations, the first two methods take about 1500 iterations to reduce the residual by about 3 orders of magnitude but overall convergence eventually stalls at $2.5 \times 10^{-7}$. The JFNK-2 solver, on the other hand, has no such problems. The improvement in the convergence rate is due to the fact that JFNK-2 intrinsically accounts for the MUSCL reconstructed states and the slope limiter in the numerically-approximated Jacobian. In terms of wall time, both JFNK-1 and JFNK-2 are much slower than the explicit Jacobian. This is because at each FGMRES iteration, JFNK performs a residual function evaluation, while the explicit Jacobian method performs a matrix vector multiplication, which is much faster. Figure 5 shows the convergence histories of the lift and drag coefficients. It should be noted that although the convergence of JFNK-1 and explicit Jacobian stall, the physical quantities actually converge.

The advantage of JFNK solver lies in the memory consumption: JFNK-2 (42.22 GB) achieves a $60 \%$ reduction over the explicit Jacobian method (106.62 GB). All simulations were carried out in parallel on 48 processors.

Table 1. Trap wing: comparison of the lift and drag coefficients

\begin{tabular}{rll}
\hline \hline Methods & $C_{L}$ & $C_{D}$ \\
\hline Experiments & 2.0468 & 0.3330 \\
Explicit Jacobian & 2.0102 & 0.3318 \\
JFNK-1 & 2.0107 & 0.3319 \\
JFNK-2 & 2.0113 & 0.3320 \\
\hline
\end{tabular}



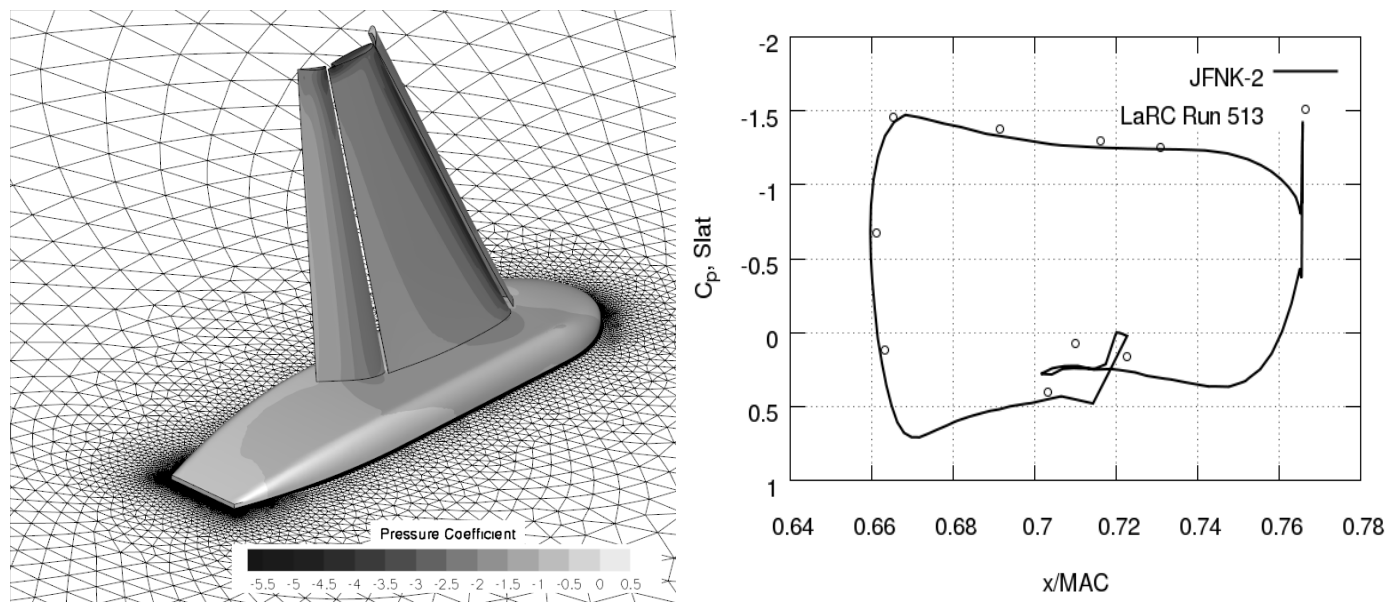

Figure 1. Trap Wing: surface pressure coefficient contours (left) and pressure coefficient distribution on the slat at the $50 \%$ spanwise station (right)
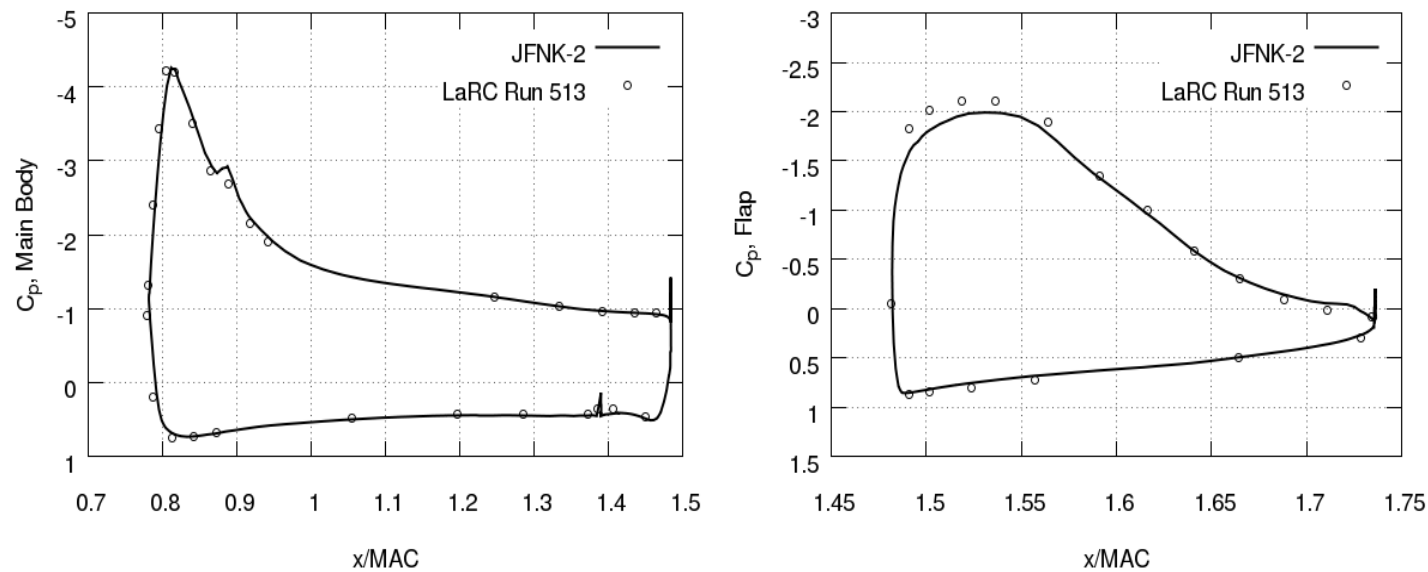

Figure 2. Trap Wing: pressure coefficient distribution over the main element (left) and flap (right) at the $\mathbf{5 0 \%}$ spanwise station


Figure 3. Trap Wing: surface shear stress magnitude contours (left) surface shear stress magnitude distribution at the $50 \%$ spanwise station (right) 

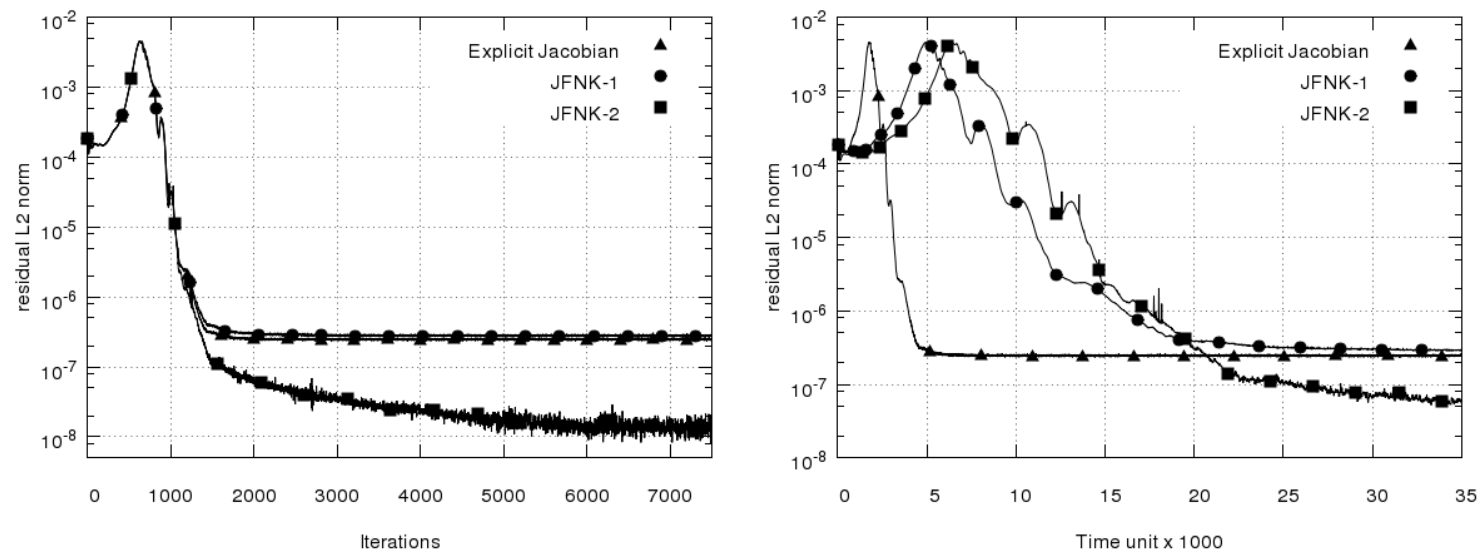

Figure 4. Trap Wing: convergence as a function of the number of iterations (left) and elapsed time (right)
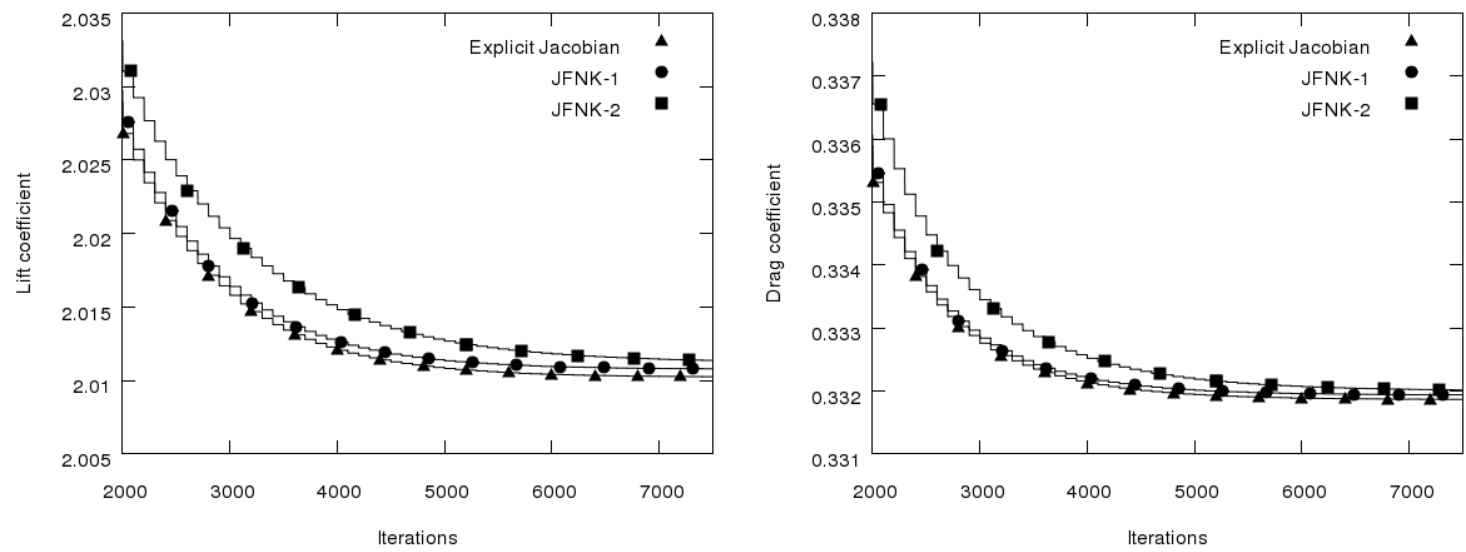

Figure 5. Trap wing: convergence history of the lift (left) and drag coefficients (right)
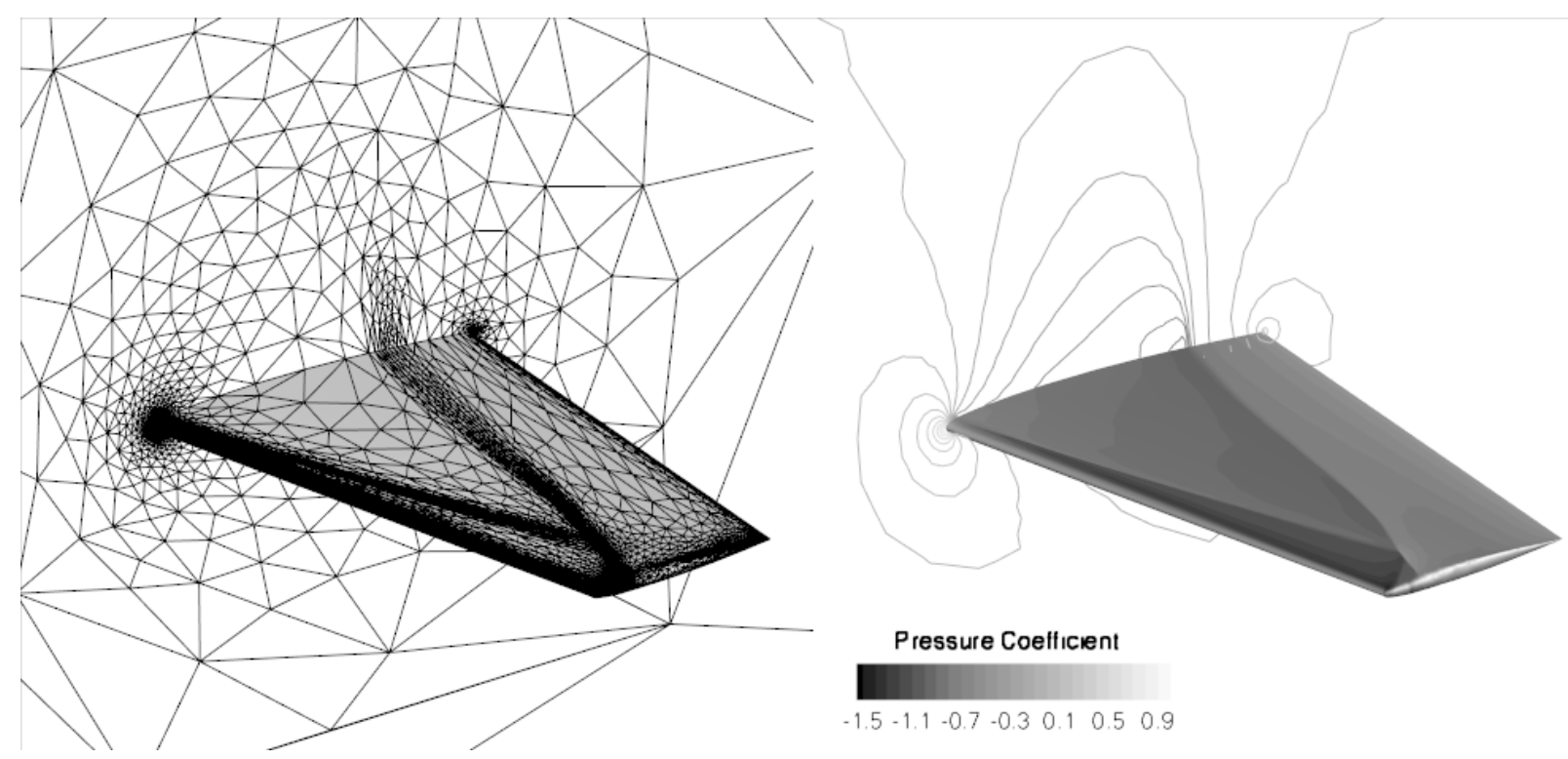

Pressure Coefficient

$\begin{array}{llllllll}-1.5 & -1.1 & -0.7 & -0.3 & 0.1 & 0.5 & 0.9\end{array}$

Figure 6. ONERA M6 wing: final adapted mesh (left), Mach contours (right) 


\section{B. Mach 0.84 inviscid Flow past the ONERA M6 Wing}

This example presents the Mach 0.84 inviscid transonic flow over the ONERA M6 wing at an angle of attack of 3.04 degrees. The initial mesh consists of 87,795 nodes and 396,412 tetrahedra. Three mesh adaptation cycles have been performed [29] using the Mach number to build the error estimator. The target number of nodes is 136,051 , the same number of nodes adopted by Luo [4]. The final adapted mesh, shown in Figure 6, consists of 127,077 nodes and 674,500 tetrahedra. The initial CFL numbers is 1 and is exponentially increased to 1,000 in 120 iterations.

Figure 6 presents the pressure coefficient on the final adapted mesh. The solution over the adapted mesh displays sharp shocks and captures the complex lambda-type shape. The validation with experimental results from [30] is shown in Figure 7, which also illustrates the positive effect of mesh adaptation on the solution. The comparison between the computational results on the final adapted mesh and the experimental results is satisfactory. The strong shock wave observed in the numerical results at mid-chord near the root of the wing is absent in the experimental results. This discrepancy is due to the lack of viscous effects in the numerical solution.

Figure 8 shows the convergence history for the explicit Jacobian with ILU (top), JFNK-2 (middle) and JFNK-1 (bottom). The time unit is defined as the average time per FGMRES iteration for JFNK-2 and it is 0.5 seconds for this test case. The cycles are labeled $0^{\text {th }}$ (original grid), $1^{\text {st }}, 2^{\text {nd }}$ and $3^{\text {rd }}$ adaptation cycle. At the end of each adaptation cycle, the solution on the previous mesh is interpolated onto the new adapted mesh to provide a faster restart. This is reflected in the convergence plot as a jump in the residual. JFNK-1 stalls on all the meshes. When it reaches the maximum number of Newton iterations, 250, it stops and moves on to the next interpolated mesh. The explicit Jacobian method stalls on the first and fourth meshes while JFNK-2 converges at each adaptation cycle. This improvement in the convergence rate is again attributed to the fact that JFNK automatically accounts for the MUSCL reconstructed states and the slope-limiter. Overall JFNK-2 takes half as many iterations as explicit Jacobian, but is about two times slower in terms of wall time. This is in contrast to Luo's observations [4], who reported that GMRES+LU-SGS (similar to JFNK-2 in this work) is about three times faster than the GMRES+ILU (similar to explicit Jacobian in this work). Several possible reasons could explain the differences. In Luo's work, the Jacobian-free condition in the GMRES solver is attained by the same methodology used in LU-SGS, which has been

illustrated in Section III.C, but in this work it is realized by JFNK. Moreover, the implementation of LU-SGS is different and the performance is certainly case-dependent. It must also be noted that in [4] GMRES is used as opposed to FGMRES, which is a more suitable choice since the preconditioner is variable. However, the current 
performance result is similar to that of Qin [31] who showed that the matrix-free solver takes more CPU time than explicit Jacobian.
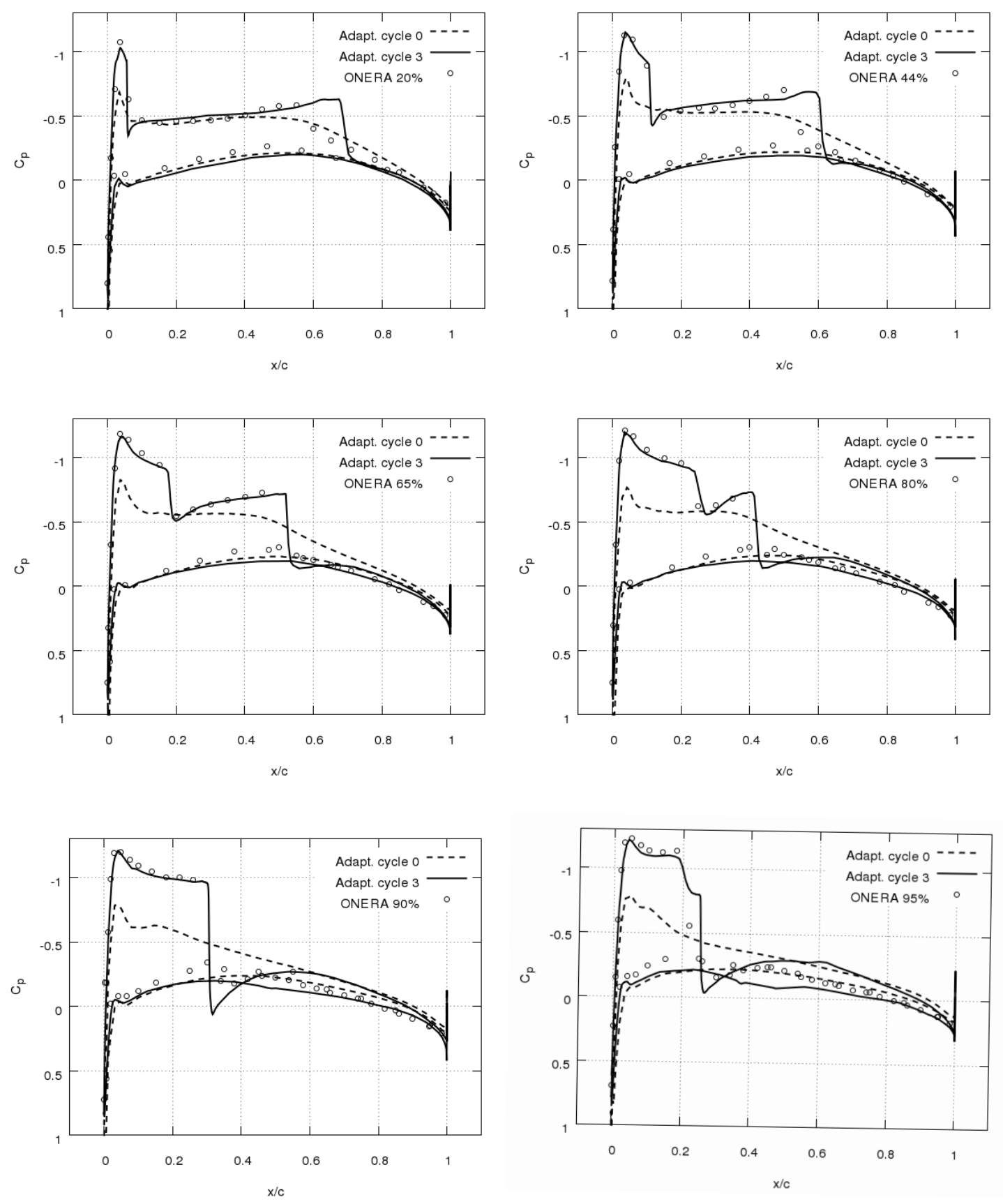

Figure 7. ONERA M6 wing: comparison of the computed surface pressure coefficients on the initial and final adapted mesh with the experimental results at the $20 \%, 44 \%, 65 \%, 80 \%, 90 \%$ and $95 \%$ spanwise stations 

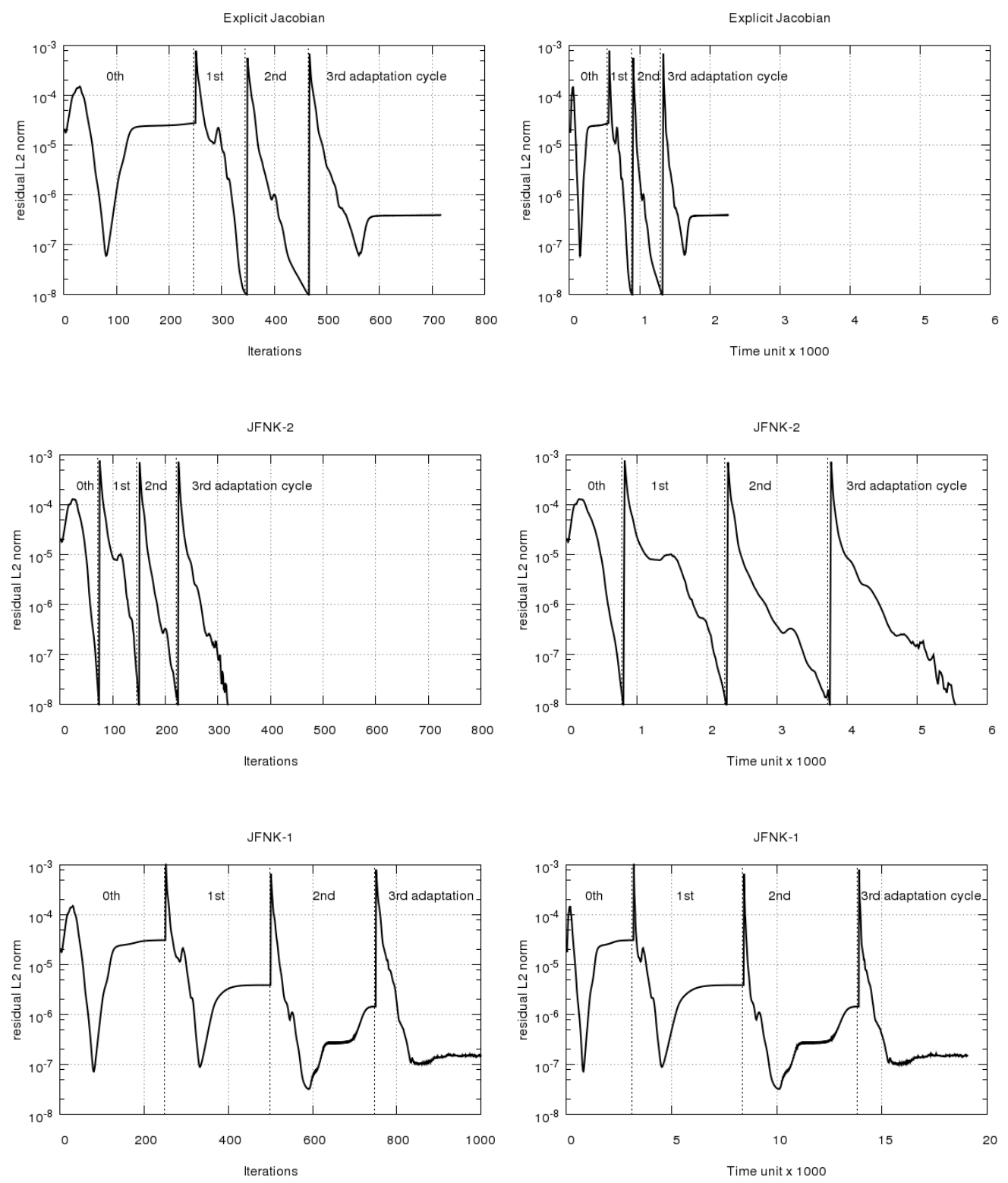

Figure 8. ONERA M6 wing: comparison of the convergence history as a function of the number of iterations (left) and time (right) between the explicit Jacobian (top), JFNK-2 (middle) and JFNK-1 (bottom)

\section{Mach 1.93 Viscous Flow past a Sphere}

This example presents the Mach 1.93 viscous flow around a sphere. The sphere radius $\mathrm{R}$ is $7.5 \mathrm{~mm}$, the freestream temperature and pressure are $294 \mathrm{~K}$ and $540 \mathrm{~Pa}$, respectively. The Reynolds number based on the sphere radius is 1750 , which is small enough to assume laminar flow. The computational grid, shown in Figure 9, consists of 300,993 nodes, 281,484 tetrahedra and 487,680 prisms. The surface of the sphere is represented by 8,128 
triangles. The near-wall region is represented by 60 layers of prisms and is approximately $3.9 \mathrm{R}$ thick. The initial CFL value of $10^{-1}$ is exponentially increased to 100 in 500 iterations.
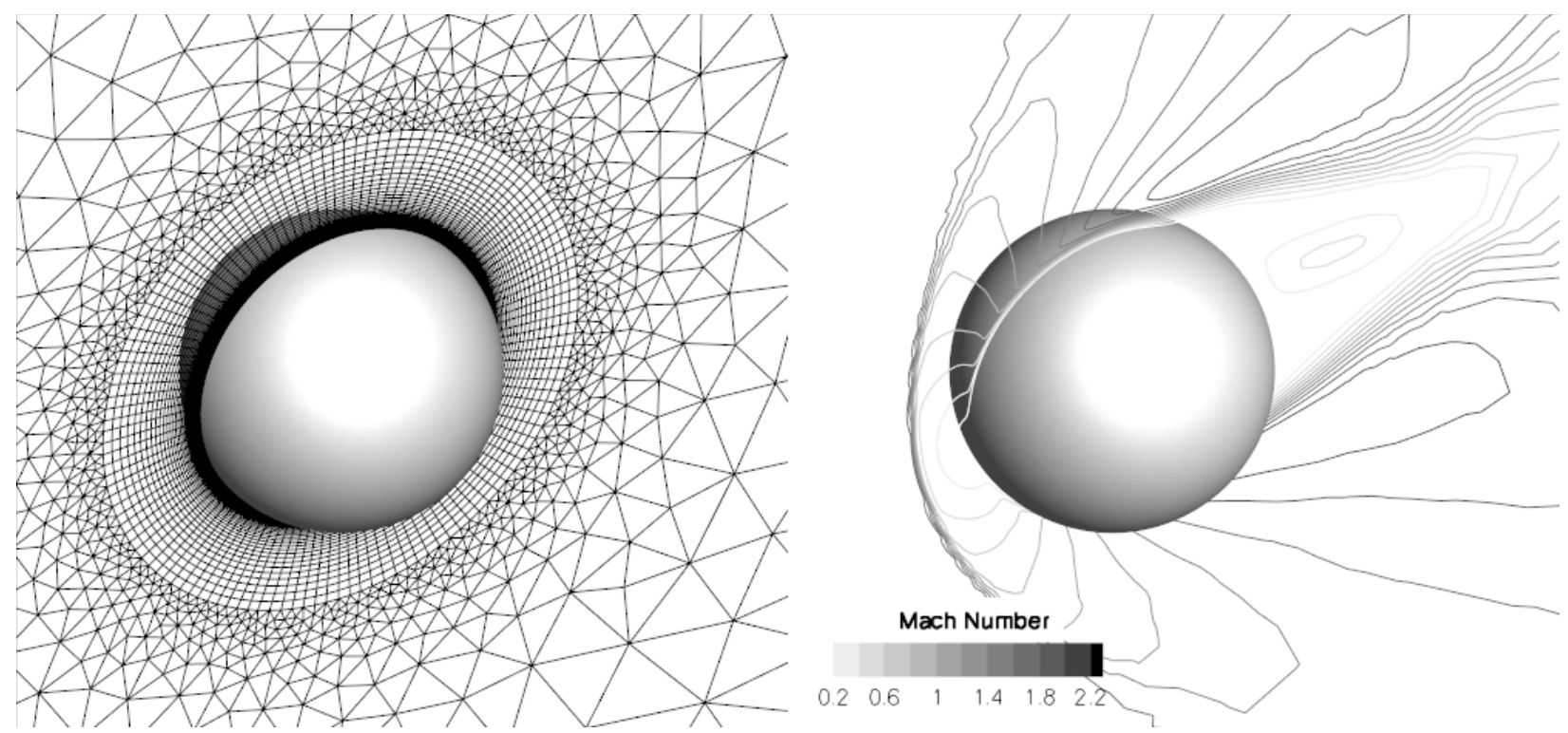

Figure 9. Mach 1.93 viscous flow over a sphere: hybrid grid (left) and Mach number contours (right)
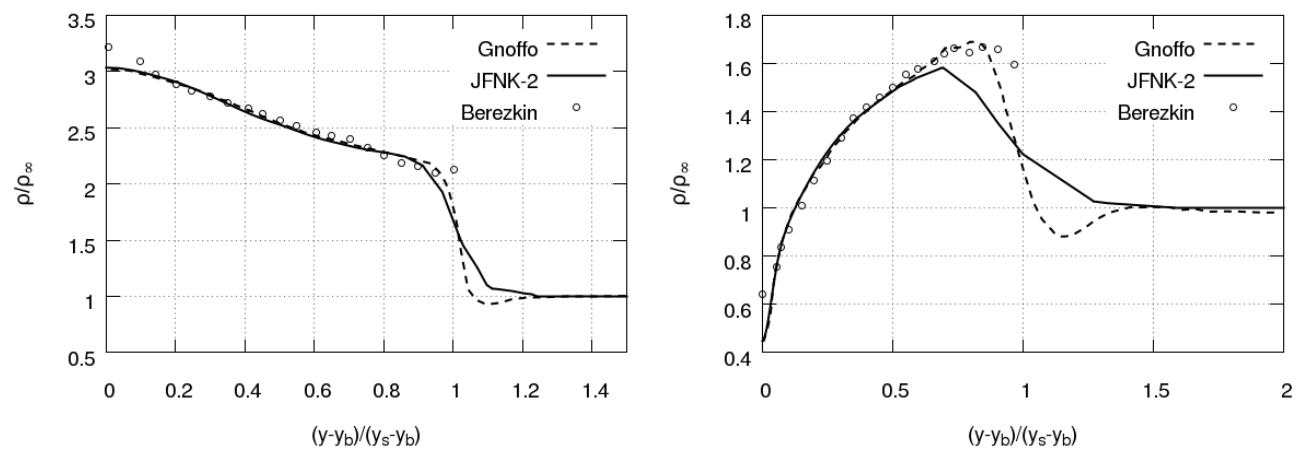

Figure 10. Mach 1.93 viscous flow over a sphere: non-dimensional density along the line normal to the axis in front of the sphere (left) and along the line normal to the sphere (right)

The Mach contours are shown in Figure 9. A bow shock appears in front of the sphere. The non-dimensional density is plotted in Figure 10 along the crosswind direction at two different locations: at the nose, $x=R$, and at the mid-section, $x=0$. The crosswind coordinate is scaled with respect to the distance between the shock location, $y_{s}$, and the boundary location, $y_{b}$. Since neither of the references provides a value for it, $y_{s}$ is measured from the solution: $y_{s}(x=0)=2.743 R$ and $y_{s}(x=R)=1.32 R$. The agreement between the solution obtained with the proposed method and the results of Gnoffo [32] is fairly good, and so is the agreement with the experiments [32]. Both the present computation and the result by Gnoffo under predict the value of the density at the stagnation point. 

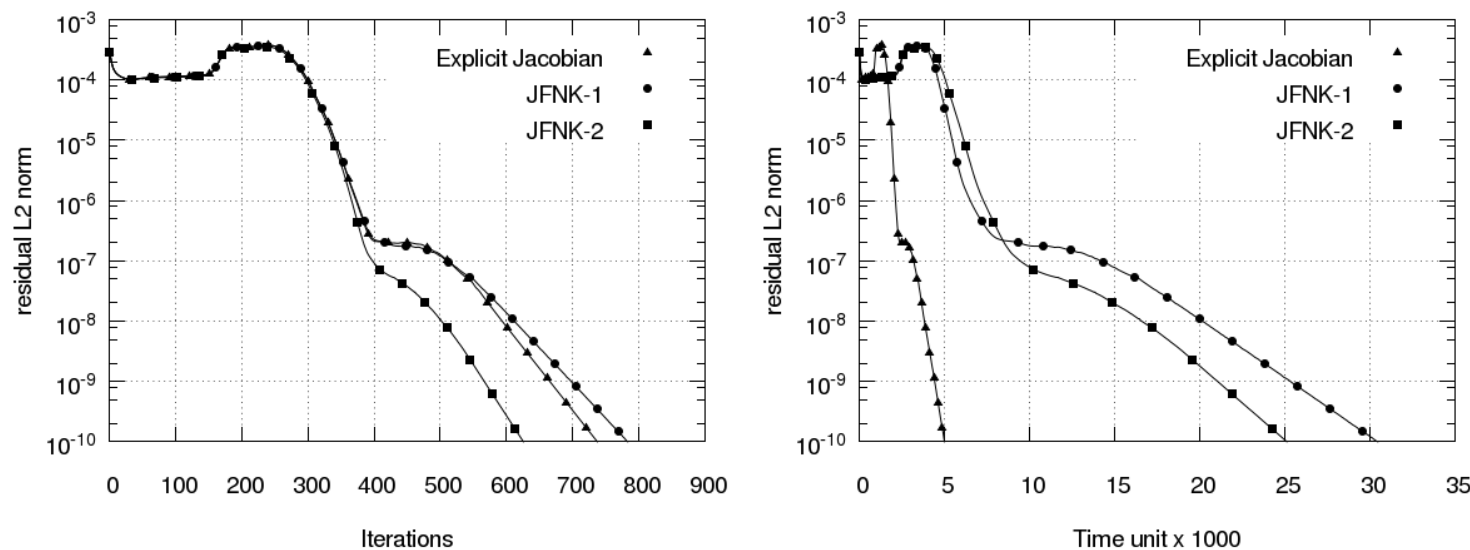

Figure 11. Mach 1.93 viscous flow over a sphere: convergence history as a function of the number of iterations (left) and time (right)

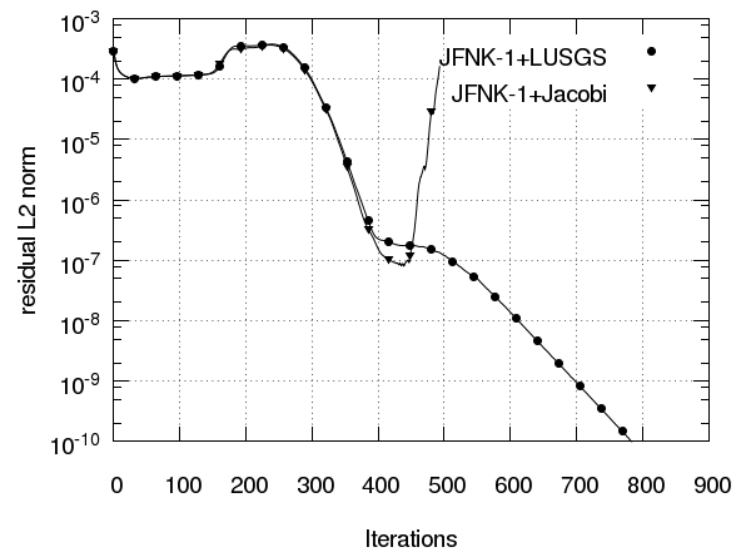

Figure 12. Mach 1.93 viscous flow over a sphere: convergence history of the LU-SGS and Jacobi preconditioners
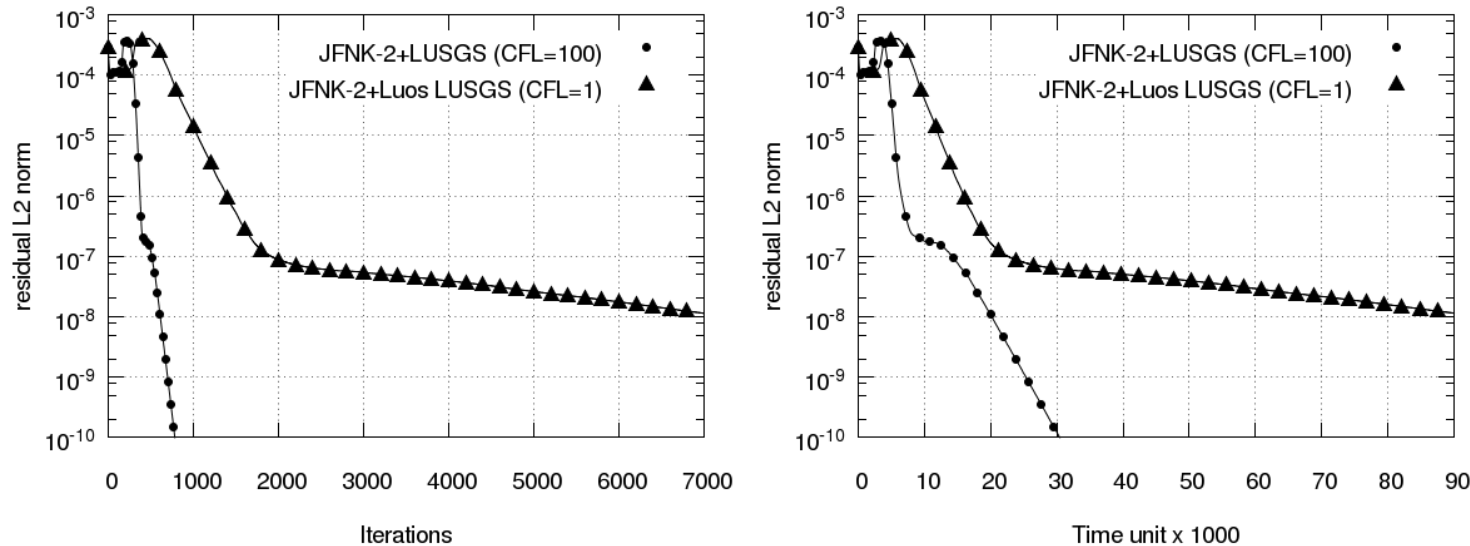

Figure 13. Mach 1.93 viscous flow over a sphere: convergence history of the LU-SGS and Luo's LU-SGS preconditioners as a function of the number of iterations (left) and time (right) 
Figure 11 shows the convergence history for the explicit Jacobian, JFNK-1 and JFNK-2. Time is defined as the average time per FGMRES iteration for JFNK-2, which is $0.48 \mathrm{~s}$ for this test case. All the residuals converge to $10^{-10}$. The advantage of JFNK-2 is in the number of Newton iterations, only 626. This is expected because JFNK-2 includes the MUSCL reconstructed states and the slope-limiter in the approximate Jacobian. In terms of wall time, JFNK-1 and JFNK-2 take about the same time. Both of them are about 6 times slower than the explicit Jacobian method. In terms of the maximum memory storage, JFNK-2 (1.48 GB) achieves a 54\% reduction over explicit Jacobian (3.18 GB). All simulations were run in parallel on 4 processors.

Figure 12 shows the comparison between JFNK-1 with LU-SGS and JFNK-1 with the Jacobi preconditioner.

In conjunction with the LU-SGS preconditioner, FGMRES convergence is achieved when the relative drop in the preconditioned residual norm is below the specified tolerance of $10^{-2}$ or a maximum number of FGMRES iterations 20, whereas for the Jacobi preconditioner the tolerance was set at $10^{-6}$ and the maximum number of FGMRES iterations was set to 200. In general, Jacobi is a less effective preconditioner than LU-SGS and thus requires more linear iterations to converge. The size of the Krylov space is 20 for both methods. Stabilization is provided by the $\mathrm{AUSM}^{+}$-up scheme with the van Albada slope limiter. Although more FGMRES iterations are allowed in the case of the Jacobi preconditioner, it still fails at around 500 Newton iteration. This suggests that the Jacobi preconditioner is, for this case, less robust than LU-SGS. Note that the chosen maximum number of linear iterations is ten times greater for Jacobi, but it is not sufficient to prevent numerical instabilities. Figure 13 shows the comparison between JFNK-2 with LU-SGS and Luo's LU-SGS preconditioner [4]. The maximum CFL is 100 for LU-SGS and 1 for Luo's LU-SGS. The cases of maximum CFLs 100 and 10 have been tested for Luo's LU-SGS, but both fail due to negative temperature, which suggests that robustness increases when the contributions from the boundaries are included and more accurate viscous fluxes are adopted.

An analysis of the effects of the relative tolerance level and maximum number of FGMRES iterations (ksp_max_it) was carried out. Two relative tolerance levels were considered, $10^{-2}$ and $10^{-6}$. The maximum number of FGMRES iterations was set to $5,10,40,80,120,160$ and 200. Figure 14 displays the results obtained with a relative tolerance of $10^{-2}$ and different values of maximum number of FGMRES iterations for the explicit Jacobian method (top), JFNK-1 (middle) and JFNK-2 (bottom). The left column shows the convergence history in terms of Newton iterations. The middle column shows the convergence history in terms of time units. The right column shows the number of FGMRES iterations in terms of Newton iterations. The parameters in Figure 15 are the same as those in 
Figure 14, except that the relative tolerance level is set to $10^{-6}$. A clear trend can be seen where the wall time typically increases with the maximum number of FGMRES iterations. However, there are exceptions with maximum number of FGMRES iterations of 5 and 10.
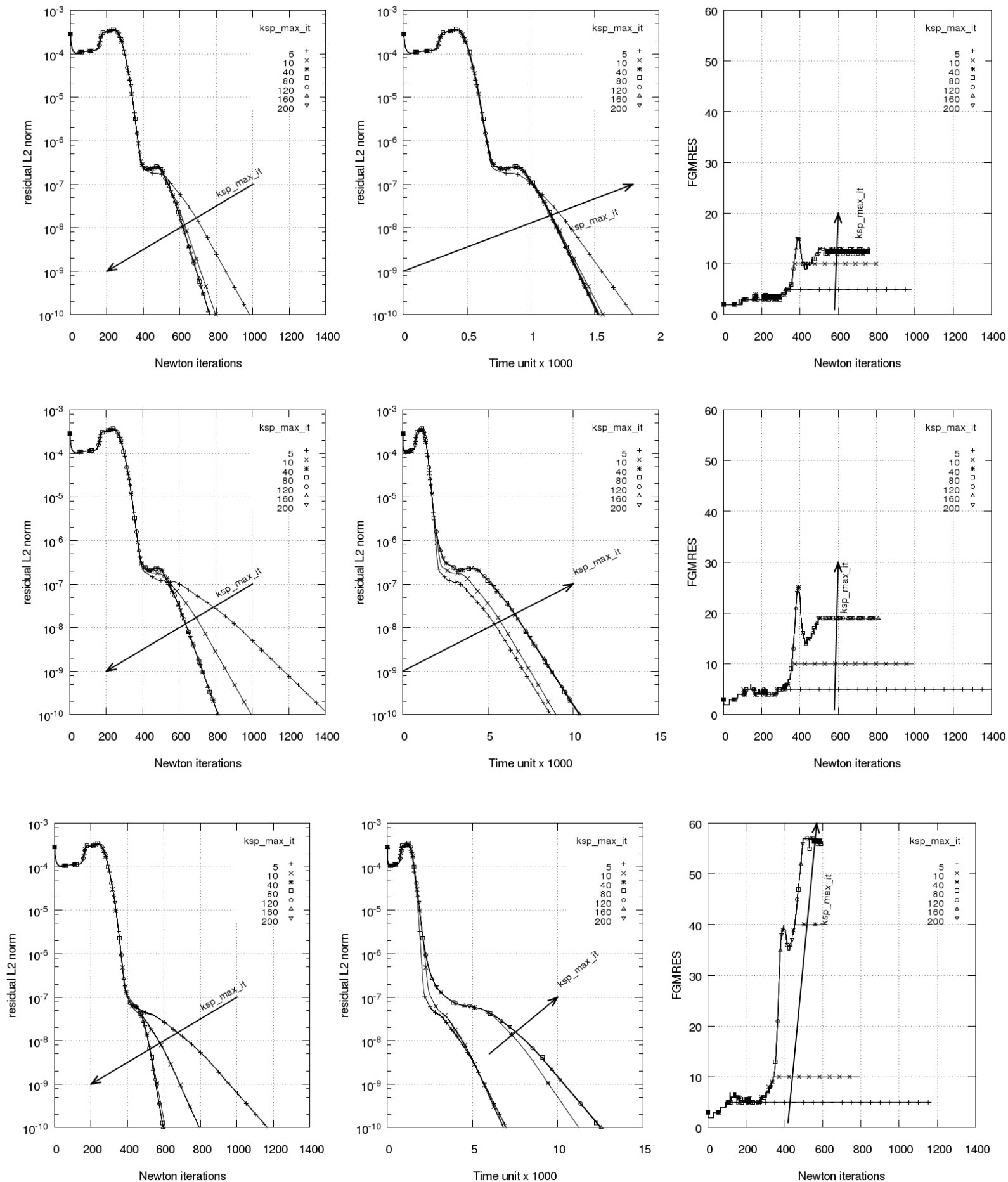

Figure 14. Mach 1.93 viscous flow over a sphere: behavior of FGMRES for the explicit Jacobian method (top), $1^{\text {st }}$ order JFNK (middle) and $2^{\text {nd }}$ order JFNK (bottom) with FGMRES relative tolerance of $10^{-2}$ 

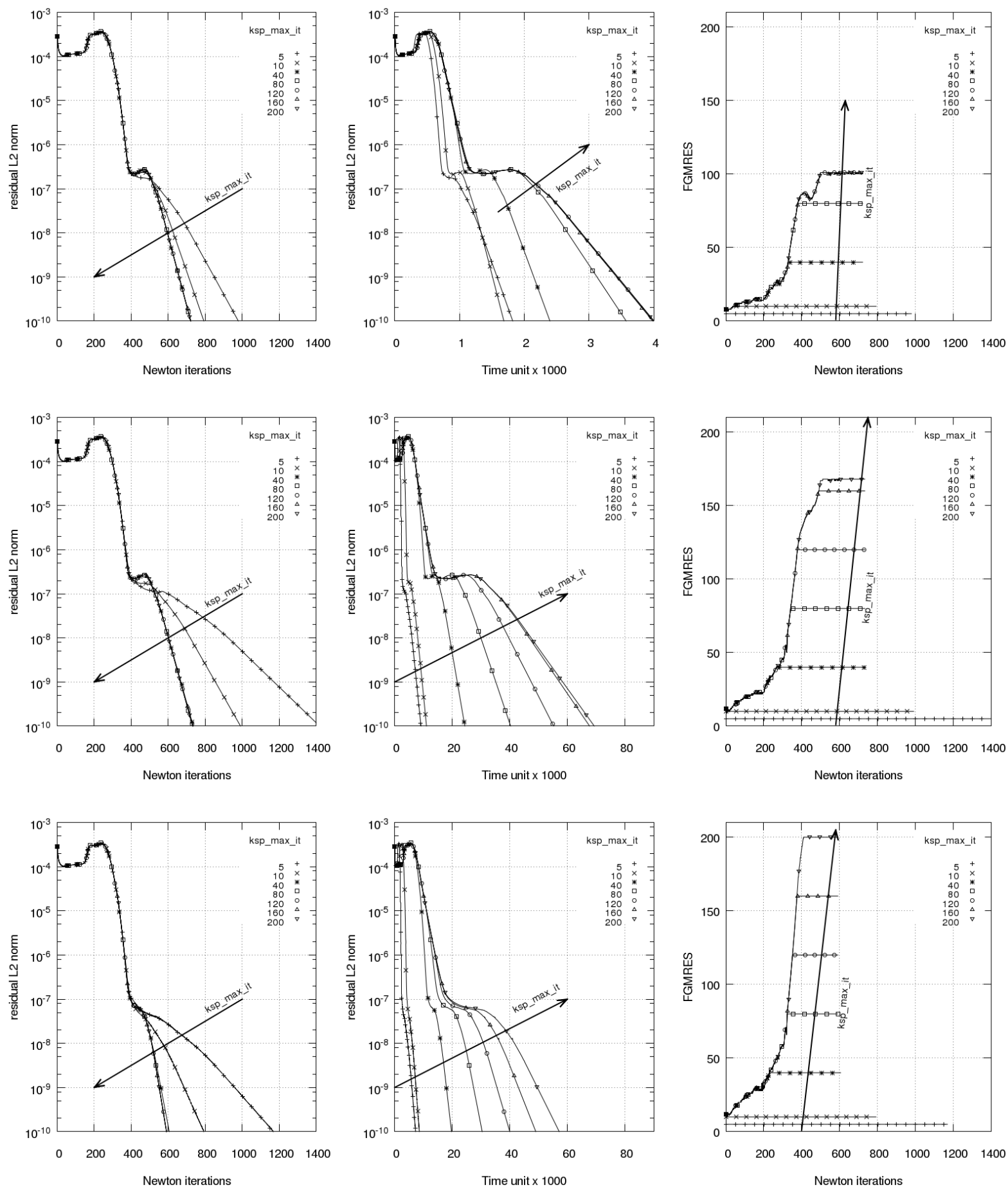

Figure 15. Mach 1.93 viscous flow over a sphere: behavior of FGMRES for the explicit Jacobian method (top), $1^{\text {st }}$ order JFNK (middle) and $2^{\text {nd }}$ order JFNK (bottom) with FGMRES relative tolerance of $10^{-6}$

The second graph in the first row of Figure 14 shows that the explicit Jacobian takes the longest time with these settings. The second graph in the third row of Figure 14 shows that JFNK-2 is slower with a maximum number of 
FGMRES iterations of 5 compared to 10. The second graph in the first row of Figure 15 shows that with lower relative tolerance levels the explicit Jacobian is faster with maximum number of FGMRES iterations of 5 and 10, however 10 is faster than 5 . The above exceptions suggest that a maximum number of FGMRES iterations of 5 is too small to ensure an appropriate convergence of the linear system.

It is also interesting to see that in the first columns of Figure 14 and Figure 15 that values of maximum number of FGMRES iterations greater than 40 do not diminish the number of Newton iterations required for convergence, but instead increase the number of FGMRES iterations (third column) and consequently dramatically increase the wall time (second column). This analysis shows that in order to achieve an optimal computational time, it is desirable to put a limit on the maximum number of FGMRES iterations and the relative tolerance in the convergence of the unpreconditioned residual norm.

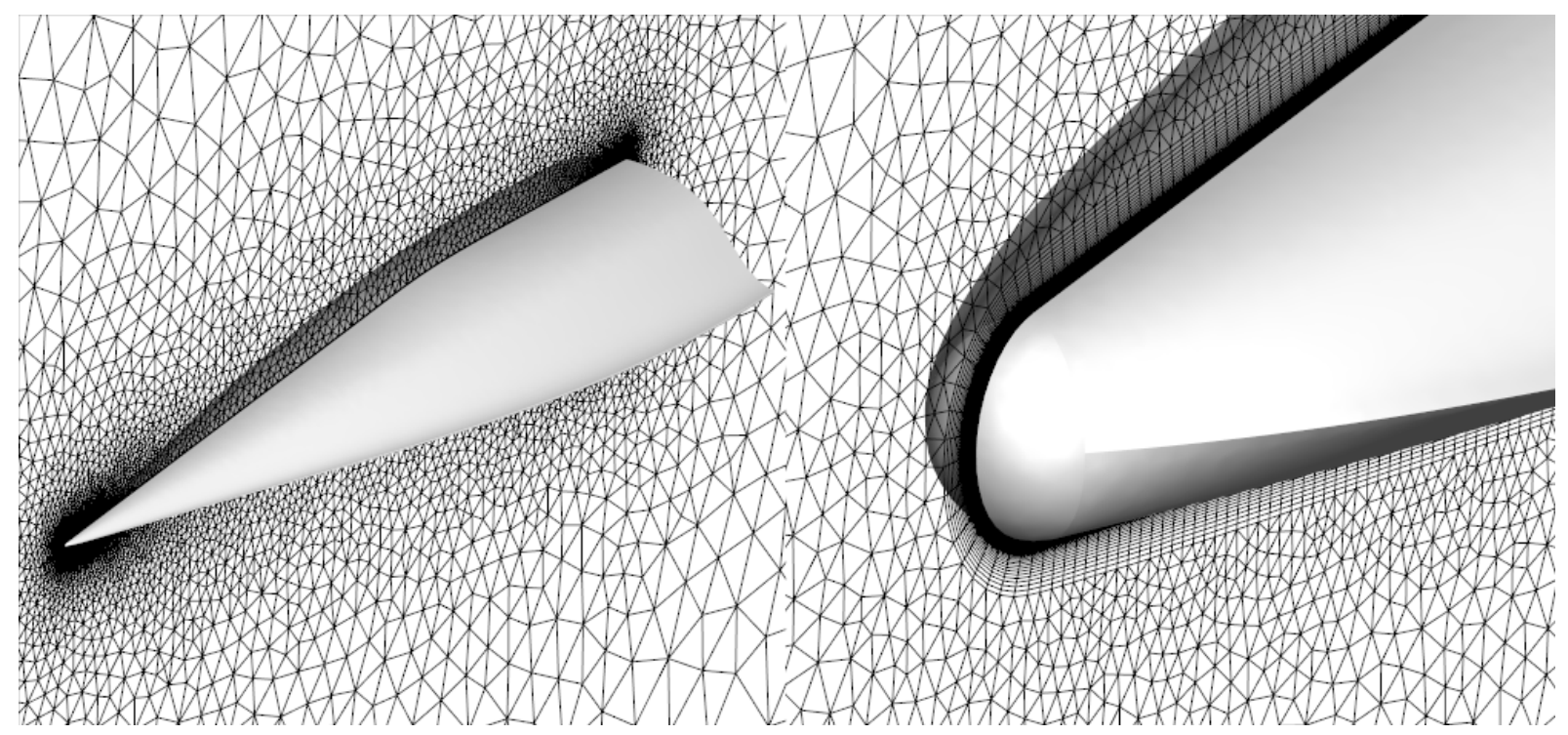

Figure 16. Mach 4.0 Viscous Flow past a Waverider: hybrid grid

\section{Mach 4.0 Viscous Flow past a Waverider}

This example presents the Mach 4.0 viscous flow around the waverider geometry shown in Figure 16. The waverider length is $3 \mathrm{~m}$, the nose radius is $0.5 \mathrm{~mm}$, and the free-stream temperature and pressure are $279.75 \mathrm{~K}$ and $8.42 \times 10^{4} \mathrm{~Pa}$, respectively. The Reynolds number based on the waverider length is $2.41 \times 10^{8}$ with an angle of attack of 1 degree. The Spalart-Allmaras turbulence model is adopted and adiabatic wall condition is specified. The computational grid consists of 4,392,286 nodes, 1,441,235 tetrahedra and 8,193,459 prisms, while the surface of the 
waverider is discretized with 151,766 triangles. The initial CFL of 0.01 is exponentially increased to 10 in 1000 iterations. All the runs take 40 FGMRES iterations at every Newton step.

The Mach and density contours are shown in Figure 17. A detached bow shock is in front of the waverider and a separation zone forms behind it. The speed-up diagram for 16, 32, 64 and 128 processors shown in Figure 18 highlights a $12 \%$ performance loss with 128 processors. The performance loss is due to the increase of the communication cost as the number of processors increase.

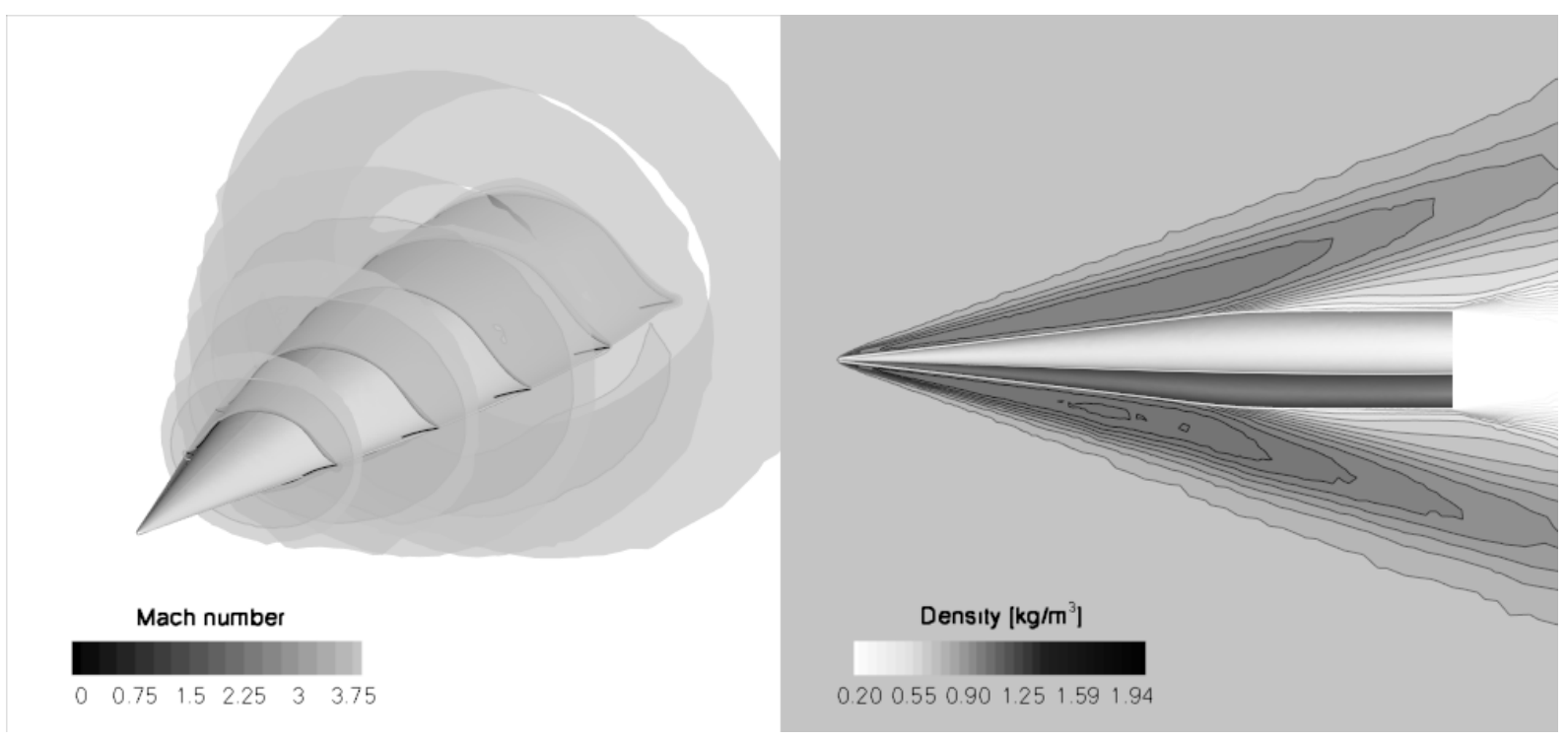

Figure 17. Mach 4.0 Viscous Flow past a Waverider: Mach number contours (left) and density contours (right)

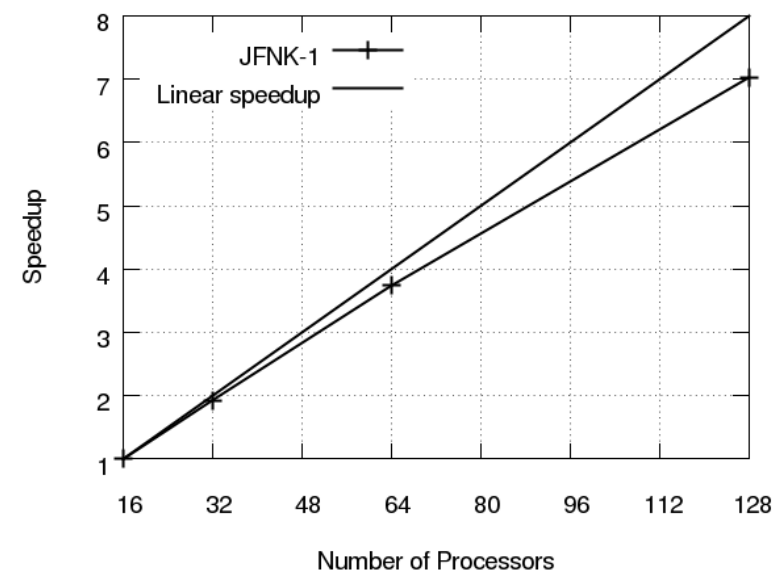

Figure 18. Mach 4.0 Viscous Flow past a Waverider: speedup diagram 

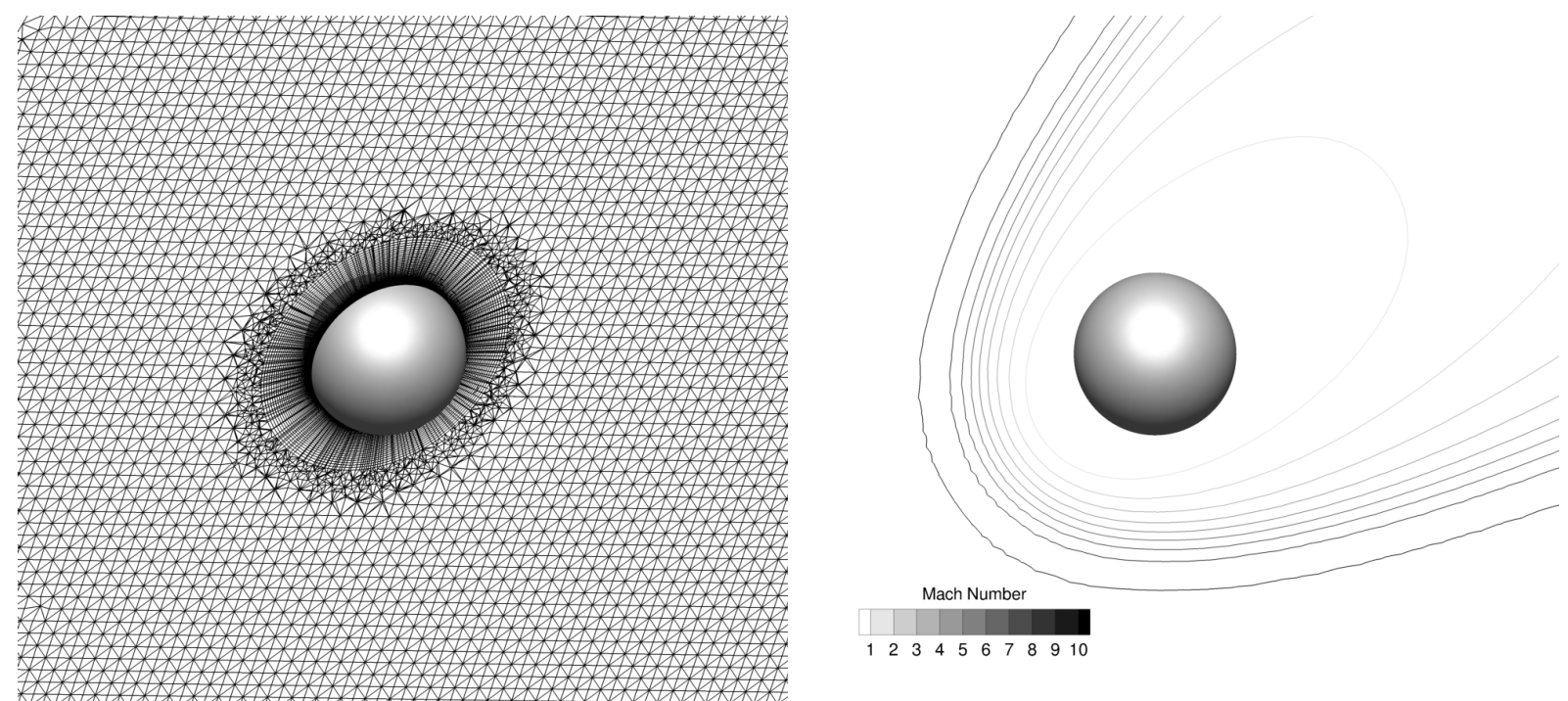

Figure 19. Mach 10.01 viscous flow past a sphere: hybrid grid (left) and Mach number (right)

\section{E. Mach 10.01 Viscous Flow past a Sphere.}

This example is the Mach 10.01 viscous flow of Nitrogen around a sphere of radius $0.1524 \mathrm{~m}$. The free stream temperature and pressure are $200 \mathrm{~K}$ and $0.0468 \mathrm{~Pa}$, respectively. The Reynolds number based on the radius of the sphere is 26.99 , which is small enough to assume a laminar flow. The computational grid, shown in Figure 19 (left), consists of 2,995,100 nodes, 16,477,103 tetrahedra and 380,800 prisms and the surface of the sphere is discretized with 234,952 triangles. The near wall region contains 40 layers of prisms and it is approximately 0.683 radii thick. The initial CFL is $10^{-5}$, exponentially increasing to $10 \mathrm{in} 3,000$ iterations. The stabilization scheme is Roe's with the van Albada slope limiter. The contours of the Mach number are shown in Figure 19 (right). As expected, at such low Reynolds number the detached bow shock in front of the sphere is significantly smeared by the viscous terms.

Figure 20 shows the convergence curves for explicit Jacobian, JFNK-1 and JFNK-2. The time unit is defined as the average time per FGMRES iteration for JFNK-2 and it is $2.7 \mathrm{~s}$ for this test case. In terms of number of Newton iterations, all solvers converge in approximately 3,200 Newton iterations. In terms of wall time, JFNK-1 is about 6 times slower than explicit Jacobian and JFNK-2 is about 4 times slower than explicit Jacobian.

In terms of maximum memory, JFNK-2 $(17.18 \mathrm{~Gb})$ achieves a $45 \%$ reduction over the explicit Jacobian method (31.64Gb). The maximum memory consumptions of JFNK-1 (17.20Gb) and JFNK-2 (17.18Gb) are nearly the same. All simulations were carried out in parallel on 48 processors. 

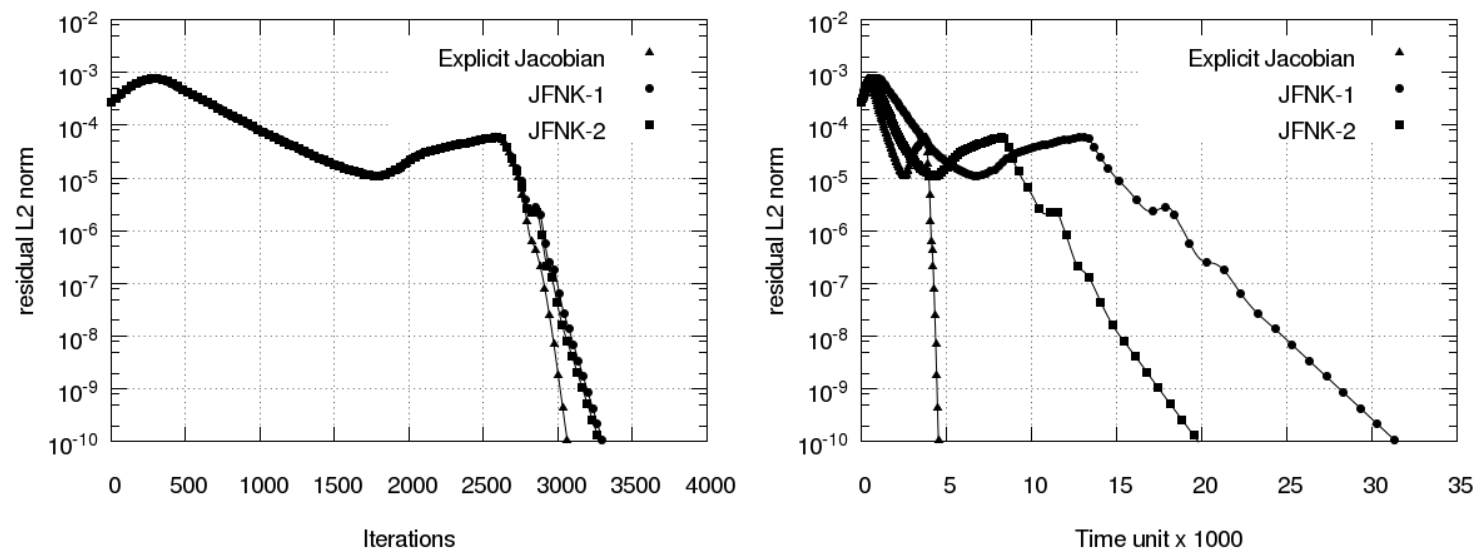

Figure 20. Mach 10.01 viscous flow past a sphere: convergence history as a function of the number of iterations (left) or time (right)

\section{Conclusion}

An accurate edge-based Jacobian-free FE solver has been developed to address low- to high-Mach viscous flows within a unified approach. This has been realized by formulating and implementing an edge-based discretization with AUSM+-up or Roe stabilization scheme, JFNK and LU-SGS preconditioner. Three-dimensional test cases are presented, with good agreement with the references.

The performance of the Jacobian-free solver is assessed. Improved LU-SGS is found to be more robust and efficient than the Jacobi preconditioner and the original LU-SGS. Comparisons between Jacobian-free method and explicit Jacobian method are carried out and the results show that the present method, despite being less performing than the explicit Jacobian, it offers relevant advantages. First, with the proposed Jacobian-free approach, the MUSCL reconstructed and slope-limited states are automatically considered. This benefit will be crucial for solving chemical and thermal non-equilibrium flows, where the transport coefficients and reaction rate coefficients are functions of the primitive variables. Second, the Jacobian-free method introduces a general framework that will allow the introduction of an arbitrary number of reactions/chemical species and non-equilibrium effects in an efficient manner. The method only needs the evaluation of the residual and does not require an analytical expression of its derivative. This is a major bonus when complex chemical and thermodynamic models are considered. Third, Jacobian-free solvers are more memory-efficient. Memory savings greater than $50 \%$ are observed for chemically non-reacting and thermal equilibrium flows and it is expected that when more complex physical phenomena are introduced and the number of chemical species and governing equations is substantially increased, savings will be much higher. 


\section{Acknowledgments}

The authors acknowledge the financial support of the NSERC-Lockheed Martin Industrial Research Chair at the McGill CFD Lab and through grant G238199 to ANSYS. We are grateful to Dr. Ned Allen, Chief Technologist, Drs. Luke Uribarri and Ben Miller from Lockheed Martin for the continuous discussions and their industrial highTRL vision. ADD COMPUTE CANADA

\section{References}

1. Lee, J.H., Basic Governing Equations for the Flight Regimes of Aeroassisted Orbital Transfer Vehicles, in 19th Thermophysics Conference. 1984, American Institute of Aeronautics and Astronautics.

2. Barbante, P.F. and T.E. Magin, Fundamentals of hypersonic flight-properties of high temperature gases. RTO AVT Lecture Series on Critical Technologies for Hypersonic Vehicle Development, 2004: pp. 5.1-5.50.

3. Moses, P.L., et al., NASA hypersonic flight demonstrators-overview, status, and future plans. Acta Astronautica, 2004. 55(3): pp. 619-630.

4. Luo, H., J.D. Baum, and R. Löhner, A Fast, Matrix-free Implicit Method for Compressible Flows on Unstructured Grids. Journal of Computational Physics, 1998. 146(2): pp. 664-690.

5. Kirk, B.S., et al., Modeling Hypersonic Entry with the Fully-Implicit Navier-Stokes (FIN-S) Stabilized Finite Element Flow Solver. Computers \& Fluids, 2014. 92(0): pp. 281-292.

6. Lani, A., An object oriented and high-performance platform for areothermodynamics simulations. Von Karman Institute for Fluid Dynamics, 2009.

7. Scalabrin, L.C., Numerical Simulation of Weakly Ionized Hypersonic Flow over Reentry Capsules. The University of Michigan, 2007.

8. Knoll, D.A. and D.E. Keyes, Jacobian-Free Newton-Krylov Methods: a Survey of Approaches and Applications. Journal of Computational Physics, 2004. 193(2): pp. 357-397.

9. Liu, L. and D.E. Keyes, Field-split preconditioned inexact newton algorithms. SIAM Journal on Scientific Computing, 2015. 37(3): pp. A1388-A1409.

10. Jameson, A. and S. Yoon, Lower-upper implicit schemes with multiple grids for the Euler equations. AIAA journal, 1987. 25(7): pp. 929-935.

11. Sharov, D. and K. Nakahashi, Reordering of hybrid unstructured grids for lower-upper symmetric gauss-seidel computations. AIAA Journal, 1998. 36(3): pp. 484-486.

12. Landau, L.D. and E.M. Lifshitz, Fluid Mechanics. 1959, Oxford: Pergamon Press.

13. Aris, R.X., Vectors, tensors and the basic equations of fluid mechanics. 2012: Courier Corporation.

14. Selmin, V., The Node-centred Finite Volume Approach: Bridge Between Finite Differences and Finite Elements. Computer Methods in Applied Mechanics and Engineering, 1993. 102(1): pp. 107-138.

15. Liou, M.-S., A Sequel to AUSM: AUSM+. Journal of Computational Physics, 1996. 129(2): pp. 364-382.

16. Roe, P.L., Approximate Riemann solvers, parameter vectors, and difference schemes. Journal of computational physics, 1981. 43(2): pp. 357-372.

17. Barth, T.J., Aspects of unstructured grids and finite-volume solvers for the Euler and Navier-Stokes equations. Notes for VKI Lecture Series, 1992: pp. 105.

18. Löhner, R. and M. Galle, Minimization of indirect addressing for edge - based field solvers. Communications in numerical methods in engineering, 2002. 18(5): pp. 335-343.

19. Selmin, V. and L. Formaggia, Simulation of hypersonic flows on unstructured grids. International journal for numerical methods in engineering, 1992. 34(2): pp. 569-606.

20. Van Leer, B., Towards the ultimate conservative difference scheme. V. A second-order sequel to Godunov's method. Journal of computational Physics, 1979. 32(1): pp. 101-136.

21. Venkatakrishnan, V., Convergence to steady-state solutions of the Euler equations on unstructured grids with limiters. Journal of computational physics, 1995. 118(1): pp. 120-130.

22. Hou, J., F. Simons, and R. Hinkelmann, Improved total variation diminishing schemes for advection simulation on arbitrary grids. International Journal for Numerical Methods in Fluids, 2012. 70(3): pp. 359-382.

23. Jameson, A., Time dependent calculations using multigrid, with applications to unsteady flows past airfoils and wings. AIAA paper, 1991. 1596: pp. 1991. 
24. Saad, Y., A flexible inner-outer preconditioned GMRES algorithm. SIAM Journal on Scientific Computing, 1993. 14(2): pp. 461-469.

25. Eisenstat, S.C. and H.F. Walker, Choosing the Forcing Terms in an Inexact Newton Method. SIAM Journal on Scientific Computing, 1996. 17(1): pp. 16-32.

26. Carpentieri, G., An adjoint-based shape-optimization method for aerodynamic design. 2009: TU Delft, Delft University of Technology.

27. Balay, S., et al. Petsc web page. 2016; Available from: http://www.mcs.anl.gov/petsc.

28. Rumsey, C.L., et al., Summary of the first AIAA CFD high-lift prediction workshop. Journal of Aircraft, 2011. 48(6): pp. 2068-2079.

29. Remaki, L., et al. On the a Posteriori Error Estimation in Mesh Adaptation to Improve CFD Solutions. in 44rd AIAA Aerospace Sciences Meeting and Exhibit. 2006.

30. Schmitt, V. and F. Charpin, Pressure distributions on the ONERA-M6-wing at transonic Mach numbers. Experimental data base for computer program assessment, 1979. 4.

31. Qin, N., D.K. Ludlow, and S.T. Shaw, A matrix - free preconditioned Newton/GMRES method for unsteady Navier Stokes solutions. International Journal for Numerical Methods in Fluids, 2000. 33(2): pp. 223-248.

32. Gnoffo, P.A., Forebody and afterbody solutions of the Navier-Stokes equations for supersonic flow over blunt bodies in a generalized orthogonal coordinate system. NASA TP-107, 1978.

33. Venkatakrishnan, V. and D.J. Mavriplis, Implicit solvers for unstructured meshes. Journal of computational Physics, 1993. 105(1): pp. 83-91 